\title{
Linking dynamical perceptual decisions at different levels of description in motion pattern formation: Computational simulations
}

\author{
DAVID F. NICHOLS and HOWARD S. HOCK \\ Florida Atlantic University, Boca Raton, Florida \\ and \\ GREGOR SCHÖNER \\ University of the Rühr, Bochum, Germany
}

\begin{abstract}
A two-level dynamical model of motion pattern formation is developed in which local motion/ nonmotion perceptual decisions are based on inhibitory competition between area V1 detectors responsive to motion-specifying versus motion-independent stimulus information, and pattern-level perceptual decisions are based on inhibitory competition between area MT motion detectors with orthogonal directional selectivity. The model accounts for the effects of luminance perturbations on the relative size of the pattern-level hysteresis effects reported by Hock and Ploeger (2006) and also accounts for related experimental results reported by Hock, Kelso, and Schöner (1993). Single-trial simulations demonstrated the crucial role of local motion/nonmotion bistability and activationdependent future-shaping interactions in stabilizing perceived global motion patterns. Such interactions maintain currently perceived motion patterns by inhibiting the soon-to-be-stimulated motion detectors that otherwise would be the basis for the perception of an alternative pattern.
\end{abstract}

Traditionally, the relationship between different levels of description in pattern formation has been thought to be hierarchical, with larger constituents of the pattern built from smaller, more location-specific ones. In contrast with this relatively static perspective, the research reported in the accompanying article by Hock and Ploeger (2006) provides evidence for the dynamical linkage between motion processes at different levels of description in motion pattern formation. In a series of psychophysical experiments, they showed that local perceptual decisions regarding whether or not motion is perceived affect the stability of the global patterns formed from the detected motions (stability is measured by the size of pattern-level hysteresis). The purposes of the present article are to demonstrate the computational plausibility of a dynamical theory of multilevel pattern formation and to highlight the importance of activation-dependent future-shaping interaction in stabilizing perceived motion patterns.

Hock and Ploeger's (2006) experiments were based on the motion quartet, a bistable stimulus for which either horizontal or vertical motion patterns are formed. Their main experimental manipulation entailed the introduction of luminance perturbations that were nonspecific with respect to the competing horizontal and vertical motion directions of the motion quartet; that is, neither direction

Correspondence relating to this article may be sent to H. S. Hock, Department of Psychology, Florida Atlantic University, Boca Raton, FL 33431 (e-mail: hockhs@fau.edu). was differentially favored by the luminance changes. Luminance perturbations that briefly eliminated the local, stimulus-initiated activation of motion detectors reduced or reversed pattern-level hysteresis when motion perception resumed. In contrast, luminance perturbations that briefly increased the local, stimulus-initiated activation of motion detectors maintained motion perception and increased pattern-level hysteresis. Finally, luminance perturbations that briefly reduced but did not eliminate the stimulus-initiated activation of motion detectors resulted in local motion/nonmotion bistability. Pattern-level hysteresis was maintained when motion continued to be perceived during the perturbation, but not when nonmotion was perceived instead.

Hysteresis effects for the motion quartet can result from future-shaping interactions, inhibitory interactions between currently stimulated motion detectors and detectors selective to orthogonal motion directions that will be stimulated in the immediate future (Hock, Schöner, $\&$ Giese, 2003). These interactions would stabilize currently perceived patterns by reducing the likelihood of motion being perceived in competing, orthogonal directions. The effects of local luminance perturbations on the size of pattern-level hysteresis were attributed by Hock and Ploeger (2006) to the activation dependence of futureshaping interactions. On the one hand, perturbations that interrupt feedforward activation destabilize perceived patterns (reducing hysteresis) by briefly eliminating futureshaping interactions. On the other hand, perturbations that increase feedforward activation increase the stabil- 
ity of perceived patterns (increasing hysteresis) by briefly strengthening future-shaping interactions.

In the present article, we develop a two-level dynamical model in which feedforward activation from local-level dynamical decisions influences pattern-level mechanisms through the activation dependence of future-shaping interactions. The results of the three experiments reported by Hock and Ploeger (2006) are quantitatively simulated, and the roles of motion/nonmotion bistability and future-shaping interactions are concretely demonstrated by simulations showing the temporal evolution of detector activation over the course of individual trials. Our computational model expands upon the dynamical model developed for the motion quartet by Hock et al. (2003) by embedding local and pattern levels of description in established properties of detectors in areas V1 and MT. An explicitly dynamical approach is necessary because of the interconnectedness of detectors in V1 and MT. Each detector is subject to the time-varying activational effects of the stimulus (fed forward from subcortical levels to V1 detectors, then fed forward from V1 to MT detectors), activation-dependent inhibitory influences from other detectors, activation-dependent adaptation effects, and random noise. All of these contributions to a detector's activation in turn change its influence on other detectors, which ultimately comes around again by affecting their influence on the detector's activation. This ongoing recycling of change reflects the state dependence of the V1/ MT detector network; that is, the evolving activation level of each detector depends on its immediately preceding activation state as well as the activation state of the detectors with which it interacts. If we assume that detectors have intrinsic mechanisms that stabilize their activation against randomly induced fluctuations, the distribution of activation over a population of interconnected detectors changes until the rate of change for each detector approaches 0 . It is only then that activation remains relatively unchanged for a sufficiently long period of time for a stable motion pattern to be formed and the pattern's attributes to be realized in perceptual experience (see, e.g., Grossberg, 1980; Hock et al., 2003; Schöner \& Hock, 1995). The dynamical model is outlined in Figure 1. Its differential equations and parameters are presented in the Appendix.

\section{THE MODEL}

\section{Area V1}

Local motion detection is accomplished in the model by directionally selective motion detectors in V1. Eight activation variables represent all the motions that are possible for the motion quartet (Figure 1). Hock, Kogan, and Espinoza (1997) have provided psychophysical evi-
A Eight Motions for the Motion Quartet

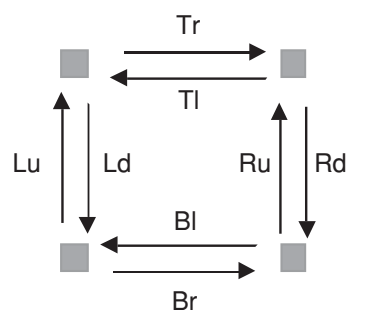

Four Sides of the Motion Quartet

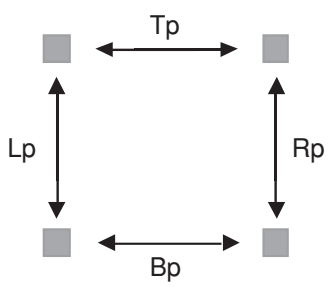

B

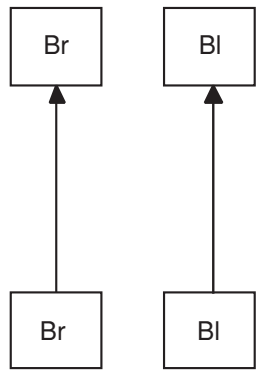

$\mathrm{Bp}$
Global-Level Pattern Formation
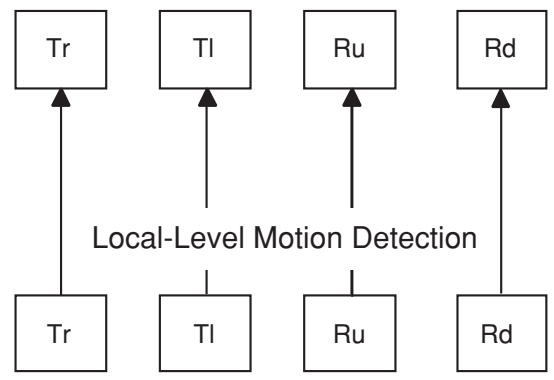

$\mathrm{Rp}$
$\mathrm{Tp}$

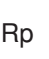

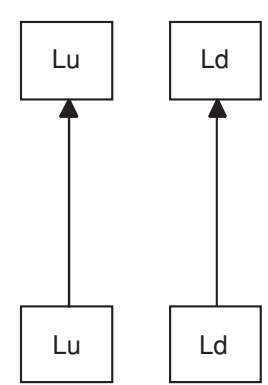

Lp
Area MT

Directionally selective motion detectors

Area V1

Directionally selective motion detectors

Detectors responsive to motionindependent spatial information

Figure 1. (A) The activation variables representing the eight detectors responsive to motion and the four detectors responsive to motion-independent spatial information along each side of the motion quartet. (B) A "flow chart" illustrating detectors in area $\mathrm{V} 1$ responsive to motion-specifying stimulus information and motion-independent spatial information, and the feed forward of motion detector activation to motion detectors in area MT with corresponding directional selectivity. Interactions among these detectors are described in the text. 
dence for hysteresis effects and spontaneous switching for single-element apparent motion. Their results are consistent with inhibitory competition between local, directionally selective motion detectors and detectors responsive to motion-independent spatial information (see also Banta \& Breitmeyer, 1985; Breitmeyer, Love, \& Wepman, 1974; Eastman \& Hock, 1999; Kulikowski, 1978). This local motion/nonmotion competition is unlikely to occur in MT because detectors there are overwhelmingly motion sensitive (Maunsell \& Van Essen, 1983). It is more likely to occur in V1, where there are detectors responsive to both motion and motion-independent spatial information (Movshon, Thompson, \& Tolhurst, 1978). Detectors with oriented receptive fields responsive to motionindependent spatial information are represented in the model by four activation variables. Each encompasses the pair of elements on one side of the motion quartet and is coupled through mutual, activation-dependent inhibitory interactions with the activation variables representing the motion directions along the same and adjacent sides of the quartet.

Motion/nonmotion competition in V1 is illustrated by a preview simulation of a trial in which a perturbation with a background-relative luminance change (BRLC) of 0 is introduced near the end of a trial in which the aspect ratio increases during successive pairs of frames (see Figure 2; the methodology for the simulation is detailed in the Method section). The square wave input in Figure 2 and elsewhere reflects the simplifying assumption that the

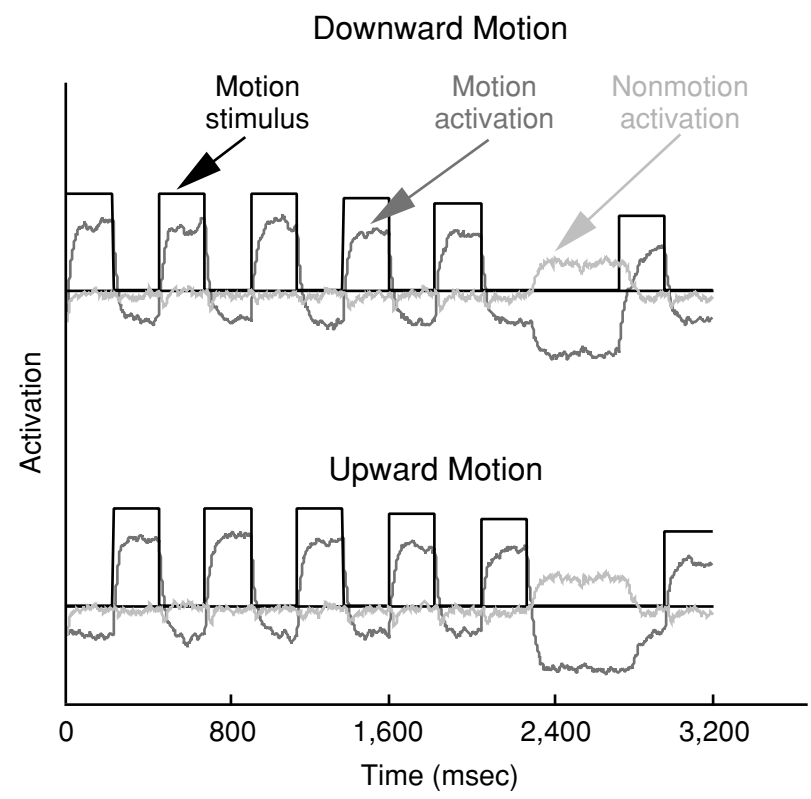

Figure 2. Preview simulation of a single trial with ascending aspect ratios (decreasing vertical activation during successive steps, beginning with the fourth step, while horizontal activation is held constant). The simulation illustrates how motion/nonmotion decisions are made in area $\mathrm{V} 1$ as a result of inhibitory competition between motion detectors and oriented detectors responsive to motion-independent spatial information. Only the detectors on one side of the quartet are included in the figure. duration of stimulus-initiated detector activation corresponds to the 200-msec duration of each frame. Stimulusinitiated detector activation decreases during successive steps for vertical motion, starting with the fourth step, and remains constant for horizontal motion. It can be seen in Figure 2 that prior to and following the perturbation, the activation of vertical motion detectors along one side of the motion quartet is above the threshold value of 0 ; that over the same time interval the activation of detectors that respond to motion-independent spatial information is below threshold; and finally, that during the perturbation frames there is a reversal in the relative activation of detectors responding to motion and detectors responding to motion-independent spatial information. Although it is not shown, this motion/nonmotion reversal is signified simultaneously along all four sides of the quartet.

\section{Area MT}

Feedforward activation from directionally selective motion detectors in V1 is directly transmitted to motion detectors in MT with corresponding directional selectivity (Movshon \& Newsome, 1996). It is in MT, not V1, that inhibitory competition takes place between detectors responsive to horizontal and vertical motion directions (Heeger, Boynton, Demb, Seidemann, \& Newsome, 1999; Recanzone, Wurtz, \& Schwarz, 1997; Snowden, Treue, Erickson, \& Andersen, 1991; Thiele, Dobkins, \& Albright, 2000). The eight activation variables representing the possible motion directions of the motion quartet are coupled by mutual inhibitory interactions between detectors with orthogonal directional selectivity. Because inhibition is activation dependent, inhibition strength is symmetrical when horizontal and vertical motion detectors are equally activated, and asymmetrical otherwise.

The strength of inhibitory coupling in the model is relatively strong for simultaneously stimulated motions with a common starting or common ending (Figure 3A; e.g., mutual inhibition between rightward motion at the top of the quartet [Tr] and downward motion on the left side of the quartet $[\mathrm{Ld}])$. These "pattern-forming" interactions account for horizontal and vertical motion never being perceived at the same time (Hock et al., 2003). The strength of inhibitory coupling is weaker in the model when the end of one motion path coincides with the start of a second motion path (Figure 3B). These "future-shaping" interactions account for the stability of the perceived horizontal and vertical motion patterns (Hock et al., 2003). For example, when rightward motion is activated at the top of the quartet (Tr) and leftward motion at the bottom (Bl), downward motion on the right $(\mathrm{Rd})$ and upward motion on the left (Lu), which will be stimulated during the immediately following frame, are suppressed. This inhibition predisposes the continued perception of horizontal motion.

\section{Perceived Motion Patterns: Hysteresis}

Rather than an "abstracted" horizontal or vertical pattern, we assume that the perceived motion pattern is embodied in the specific activation states of directionally selective motion detectors in MT. This is illustrated in Fig- 
A

Pattern-Forming Interactions
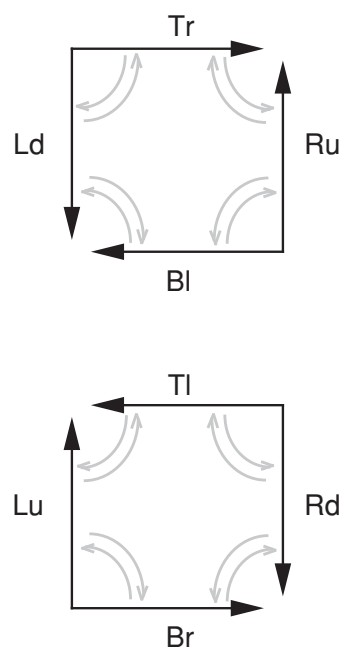

B

Future-Shaping Interactions
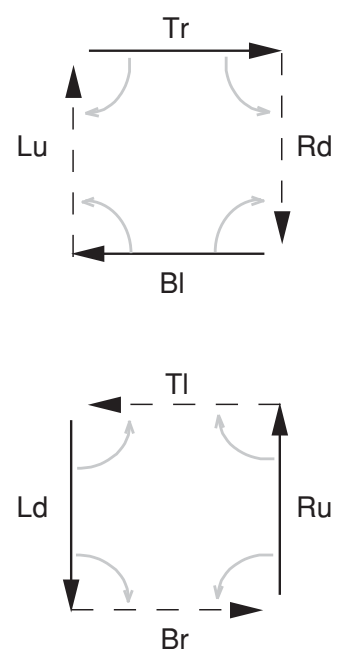

Figure 3. (A) Pattern-forming interactions for the motion quartet entail mutual inhibition between simultaneously stimulated motion directions. One or the other of the motion directions is perceived. (B) Future-shaping inhibitory interactions for sequentially stimulated motion directions. In these examples, the continued perception of a motion pattern perceived during frame $\boldsymbol{n}$ (indicated by solid arrows) is predisposed by the inhibition of competing motion directions that will be stimulated during frame $n+1$ (indicated by broken arrows).

ure 4 by the preview of a simulated trial with ascending aspect ratios (decreasing stimulus-initiated activation of vertical motion detectors) from the no-perturbation condition. In the preview simulation, the activation of each MT detector stabilizes following a frame change near a value that is either above or below threshold. These activation values constitute the stable fixed points of the detectors during that frame. It can also be seen that periodic backand-forth flow of activation occurs over the same path (e.g., alternating up/down motion as the two directions are alternately stimulated on the left side of the quartet) and, corresponding to actual perception, activation is closely coupled for detectors on opposite sides of the quartet. Vertical motions along the left and right sides of the quartet are stabilized at above-threshold activation levels early in the simulated trial, and at subthreshold activation levels later in the trial (when the perception of the horizontal motion pattern is signified). An absence of splitting also corresponds to findings from actual perception; that is, horizontal and vertical motion detectors are never simultaneously stabilized at above-threshold levels.

Alternation between two vertical motion patterns therefore is signified early in the trial, one pattern embodied in the activation of downward motion on the left and upward motion on the right side of the quartet, and the other in the activation of upward motion on the left and downward motion on the right. A switch from perception of a vertical motion pattern to perception of a horizontal motion pat-

tern did not occur until the 16th frame, when the nominal stimulus-initiated activation values were 14 for horizontal motion and 12 for vertical motion. Thus, the continued perception of vertical motion patterns is signified beyond the point at which stimulus-initiated activation values favor the perception of horizontal motion patterns, reflecting the presence of hysteresis. This embodiment of pattern perception and pattern switching in the activation of MT motion detectors is consistent with Sterzer, Eger, and Kleinschmidt's (2003) evidence that perceptual switches for the motion quartet are accompanied by changes in fMRI activity in the vicinity of area MT.

\section{METHOD}

\section{Equations}

The simulations are based on 20 coupled differential equations that describe the time-varying state of variables representing detectors responsive to motion-specifying stimulus information and detectors responsive to motion-independent stimulus information (see Appendix). Eight equations describe the changing activation state of V1 motion detectors, and 8 describe the changing activation state of the MT motion detectors that receive their input from V1 detectors with corresponding directional selectivity. (Strictly speaking, the perceived motion direction is established by local ensembles of motion detectors with different directional selectivity; see W. Kruse, Dannenberg, Kleiser, \& Hoffmann, 2002.) Four equations describe the changing activation state of $\mathrm{V} 1$ detectors responsive to motionindependent spatial information on each side of the quartet. The differential equation for each of the 20 activation variables includes terms representing (1) stimulus-initiated activation, (2) adaptation, (3) interactive coupling with other activation variables, and (4) random noise. The effects of adaptation for each of the 20 activation variables are accounted for by the changing state of 20 additional variables. Each detector's activation decreases as a result of adaptation in proportion to its current activation level, but on a much slower time scale $(2,000 \mathrm{msec})$ than the rate of change of the activation variables $(20 \mathrm{msec})$.

\section{Perceptual Thresholds and No-Stimulus Resting Levels}

The activation level constituting the threshold for perception was set at 0 , and the no-stimulus resting level was set at -5 for each V1 detector and -3 for each MT detector. Perception was signified when stimulus presentation resulted in the stabilization of activation at suprathreshold levels. Because no-stimulus levels are below threshold, activation must first pass through a range of subthreshold values, allowing detector interactions to begin at subthreshold activation levels.

\section{Stimulus-Initiated Motion Detector Activation}

The values selected for the threshold and resting levels constrain the lower end of the range of values for stimulus-initiated detector activation. In the experiments reported by Hock and Ploeger (2006), stimulus-initiated detector activation depended on the aspect ratio of the motion quartets, which varied from 0.5 to 2.0 in steps of 0.25 . The vertical interelement distance varied while the horizontal interelement distance was held constant. Stimulus-initiated motion detector activation varies inversely with interelement distance (Gilroy, Hock, \& Ploeger, 2001), so the smallest activation value (for the largest vertical distance) was set at 11 for the simulations. ${ }^{1}$ Ignoring the effects of interaction and adaptation for the moment, activation would then stabilize at 6 in V1 $(11-5)$ and at 3 in MT $(6-3)$. This leaves "room" for motion detector activation to remain above threshold, even after it is reduced by adaptation and inhibitory interaction with other detectors (only positive values of V1 activation are fed forward to MT). Values of vertical detector activation selected for 


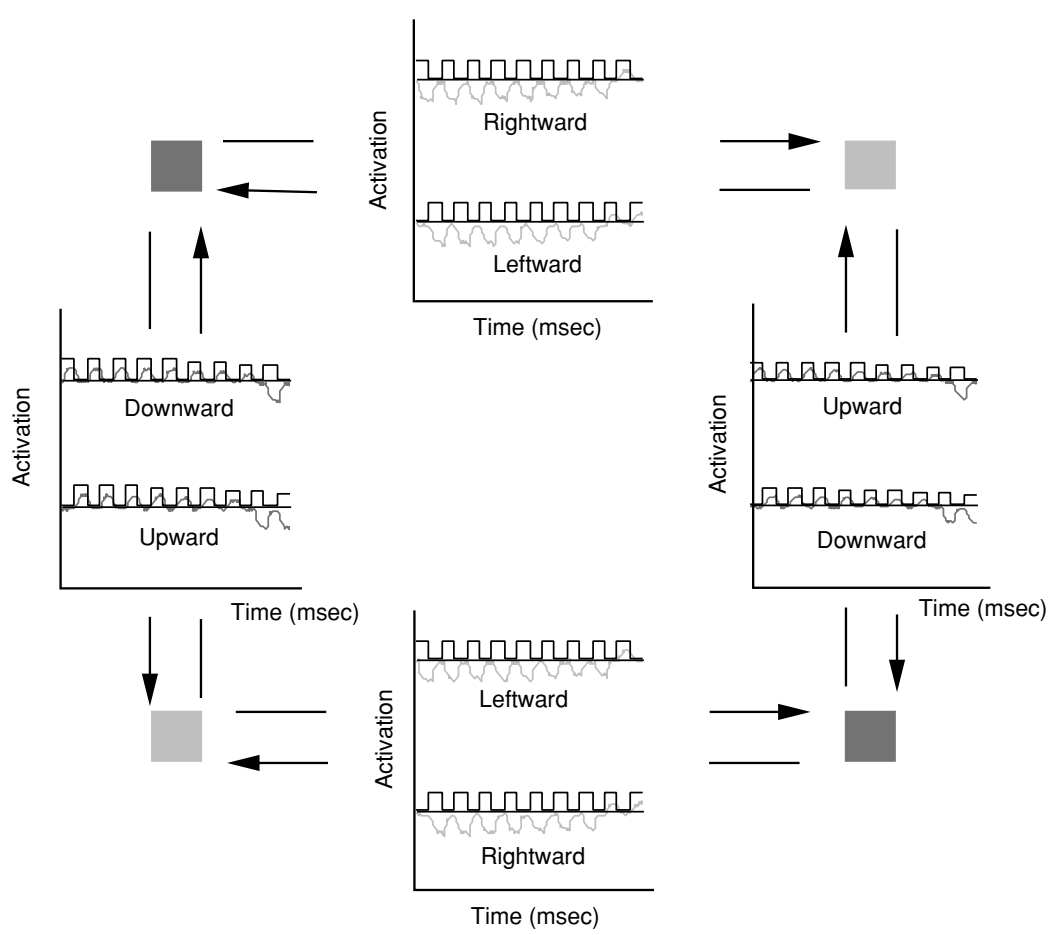

Figure 4. Preview simulation of a single trial with ascending aspect ratios (decreasing stimulus activation of vertical motion detectors during successive steps, beginning with the fourth step, while horizontal activation is held constant) illustrating how perceived motion patterns are embodied in the activation of directionally selective motion detectors in area MT. Inhibitory competition among detectors with orthogonal directional selectivity results in pattern formation (either horizontal or vertical motions are perceived, never both at the same time) and pattern stability (the vertical motion patterns formed early in the trial persist despite changes in stimulus-initiated activation that favor the perception of the horizontal motion patterns).

smaller interelement distances (thus, smaller aspect ratios) increased in steps of 1 from 11 up to 17. The stimulus-initiated activation for horizontal motion was set at 14 (the horizontal interelement distance doesn't change).

\section{Adaptation}

There is substantial neurophysiological evidence for adaptation affecting the response of motion detectors in both V1 (Giaschi, Douglas, Marlin, \& Cynader, 1993; Vautin \& Berkley, 1977) and MT (Huk, Ress, \& Heeger, 2001; Kohn \& Movshon, 2003; Priebe, Churchland, \& Lisberger, 2002; Tolias, Smirnakis, Augath, Trinath, \& Logothetis, 2001). In addition, there is psychophysical evidence with apparent motion stimuli for the adaptation of detectors responsive to motion-specifying stimulus information (Anstis, Giaschi, \& Cogan, 1985; Hock, Nichols, \& Espinoza, 2004), as well as adaptation of detectors responsive to motion-independent spatial information (Hock et al., 2004). Variables representing the effects of adaptation are therefore coupled with each of the V1 and MT activation variables. Because the adaptation effects in Hock and Ploeger's (2006) experiments are relatively short term, they are assumed to entail changes in response gain (i.e., adaptation-induced reductions in activation are proportional to the current level of activation) in both V1 and MT. (Changes in contrast gain, which require longer term adaptation, are likely to occur in V1 but not in MT; see Kohn $\&$ Movshon, 2003.) The nonlinear adaptation function illustrated in Figure 5A, which takes the form of a Naka-Rushton nonlinearity (Naka \& Rushton, 1966), characterizes the activation dependence of adaptation (i.e., the greater the activation of a detector, the pro- portionally greater the adaptation-induced decrease in activation; Giaschi et al., 1993).

\section{Motion/Nonmotion Decisions}

The interactions through which detectors are coupled in the model also take the form of a Naka-Rushton nonlinearity (see Wilson, 1998). The steep interaction function illustrated in Figure 5B characterizes the inhibitory interaction between detectors responsive to motion and detectors responsive to motion-independent spatial information. It reflects the assumption that little subthreshold interaction takes place in V1, so the stimulus-initiated activation of V1 motion detectors is either suppressed or gated through to MT essentially undiminished. The stimulus-initiated activation values of detectors responsive to motion-independent spatial information for pairs of elements on the sides of the quartet increase in an inverse relationship with vertical interelement distance (like motion detector activation), but from 9.8 to 10.6 in steps of 0.1 . The activation for pairs of elements at the top and bottom of the quartet was 10.3. With these values, motion always prevails in the motion/nonmotion competition when there are no luminance perturbations, but local decisions in favor of nonmotion could occur when luminance perturbations reduce motion detector activation.

\section{Motion/Motion Decisions}

The more sloped interaction function illustrated in Figure 5C characterizes the inhibitory interaction between MT motion detectors with orthogonal directional selectivity. The parameter values determining the shape of this function were critical for the simula- 
A Adaptation: Areas V1 and MT

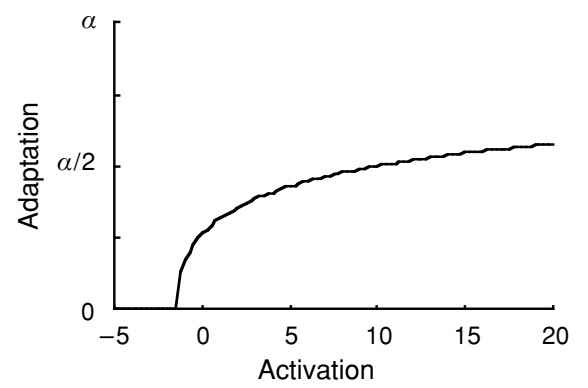

B Detector Interactions: Area V1

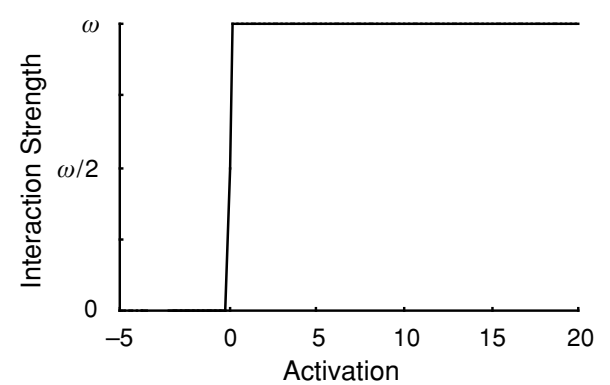

C Detector Interactions: Area MT

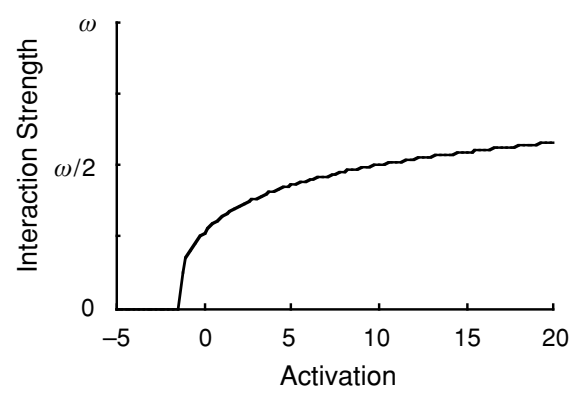

Figure 5. (A) The nonlinear Naka-Rushton (1966) function characterizing how adaptation-induced reductions in activation depend on a detector's current level of activation (scaled by the parameter $a$ ). The greater the activation of a detector, the greater the reduction in activation that results from adaptation. (B) The Naka-Rushton functions characterizing activationdependent inhibitory interactions among detectors responsive to motion-specifying stimulus information and detectors responsive to motion-independent spatial information in area $\mathrm{V} 1$, and (C) among motion detectors with orthogonal directional selectivity in area MT. (In both cases, interaction strength is scaled by the parameter $\omega_{\mathrm{s}}$ for pattern-forming interactions and $\omega_{\mathrm{n}}$ for future-shaping interactions.) The parameter values of the NakaRushton equations that determine the shapes of the adaptation and interactions function are in the Appendix.

tion of the experimental results. Two features are important. The first is that the inhibitory influence of a motion detector on orthogonal motion detectors increases rapidly with the inhibition-initiating detector's activation while this activation is still below the threshold level required for perception. This feature reflects evidence that one motion detector can inhibit another despite its own activation being subthreshold for perception (Hock, Schöner, \& Hochstein, 1996; Levinson \& Sekuler, 1975) and prevents the model from signifying the simultaneous perception of horizontal and vertical motion.
If the interaction function were shifted such that there were no subthreshold inhibitory effects between orthogonal detectors, the stimulus-initiated activation for both horizontal and vertical motion would rise above the threshold level for perception, inappropriately signifying the perception of splitting motion, before competitive interactions pushed one or the other below threshold. The second important feature of the interaction function in Figure 5C is that the inhibitory influence of a motion detector on orthogonal motion detectors gradually increases as the detector's activation stabilizes at greater suprathreshold levels. The graduated interaction function is necessary in order to account for different levels of feedforward activation affecting the strength of future-shaping interactions (see Figure 13). In general, the magnitude of the stimulus input and the strength of the decision-making interactions that suppress one of two competing motion directions must be similar in magnitude. If the stimulus-initiated activation of motion detectors were substantially greater than 17 , and the strength of inhibitory coupling remained the same, the activations of horizontal and vertical motions both would stabilize at suprathreshold levels, and perceptual "splitting" would be inappropriately signified in the simulations.

\section{Experimental Design}

The modified method of limits used in the reported experiments (Hock \& Ploeger, 2006) was directly implemented in the simulations. Separate ascending and descending trials varied with respect to the endpoint activation value for the vertical motion detectors (the equivalent of the endpoint aspect ratio in the experiments). In Hock and Ploeger's experiments, luminance perturbations entailed increases or decreases in the BRLC of each element during the nextto-last pair of frames, irrespective of the aspect ratio of the motion quartet during those frames. The BRLC is determined by dividing the luminance change for the element by the difference between the element's average luminance and the luminance of its background (Hock et al., 1997). Corresponding perturbations were introduced into the simulations by increasing or decreasing the stimulus activation values for vertical and horizontal motion during the next-to-last pair of frames, irrespective of the aspect ratio during those frames. Changes in pattern stability (and thus, hysteresis) therefore were expected at all aspect ratios for which there was a perturbation. The size and direction of these changes in luminance/activation distinguished among both the three experiments and their simulations. ${ }^{2}$

\section{Procedure}

The stochastic differential equations in the Appendix are integrated using the forward Euler method, with noise scaled by the square root of the 1-msec step size (Kloeden \& Platen, 1999). The activation state for each V1 and MT detector was calculated once per millisecond in the simulations. Whether or not the perception of a particular motion is signified during a frame was determined by categorizing activation as above or below threshold for each 1-msec sample and summing the number of above-threshold instances within a sliding 60 -msec "window" (through a convolution operation). Motion perception for a particular direction was signified for MT motion detectors when $90 \%$ of the instances (54 of 60 ) were above threshold in any of the $60-\mathrm{msec}$ windows, beginning $30 \mathrm{msec}$ after the onset of each 200-msec frame and ending $30 \mathrm{msec}$ after the offset of the frame. (The 30-msec shift accommodates the model's 20 -msec time scale of activation change, initially at V1, then at MT.) Nonmotion was signified if this criterion was not met for any of the motion directions stimulated during a frame.

When the perception of a motion pattern is signified for the quartet, motion is signified for two directions on opposite sides of the quartet (e.g., rightward on the top and leftward on the bottom), and nonmotion is signified for the other two directions stimulated during that frame (downward on the left and upward on the right). "Coupling failure" would be indicated if motion perception were signified for only one of the motion directions stimulated during a frame, but not the parallel motion on the opposite side of the quartet. 
The very rare trials for which this occurred were discarded from the simulations. If they occurred for the motion quartet in reality, their occurrence also would be very rare.

A perceptual switch is signified if the initially formed motion pattern (e.g., horizontal motions on the top and bottom) is replaced by an alternative motion pattern (e.g., vertical motions on the left and right) at any time during the simulated trial. Horizontal and vertical motions are not perceived simultaneously for the motion quartet, so "splitting failure" would be indicated if motion perception were signified simultaneously for horizontal and vertical motions stimulated during the same frame (e.g., leftward at the top and downward on the right). If this had occurred (it never did), such trials would also have been discarded from the simulation.

\section{RESULTS}

Although the effects of the perturbations on hysteresis were consistent for all 4 participants in each of the three experiments reported by Hock and Ploeger (2006), there was substantial variation in the absolute size of each participant's hysteresis effects. For this reason, the simulations of each experiment were based on the average of the results for the individual participants. Parameter values, which are listed in the Appendix, were kept constant for the simulations of all three experiments. As in Hock and Ploeger, hysteresis effects were quantified by fitting cumulative normal distributions to both the averaged empirical results and their simulations. The size of each hysteresis effect $(H)$ was determined by subtracting the $50 \%$ threshold for descending trials (the endpoint aspect ratio for which there was a switch from a horizontal to a vertical motion pattern for half of the trials) from the 50\% threshold for ascending trials (the endpoint aspect ratio for which there was a switch from a vertical to a horizontal motion pattern for half of the trials).

\section{Experiment 1}

In this experiment, the luminance perturbation reduced the BRLC from 0.8 to 0 during the next-to-last display cycle. This was implemented in the model by reducing the stimulus-initiated activation of motion detectors to 0 . All four elements remained visible (their average luminance value didn't change), so there was no reduction in the stimulus-initiated activation of detectors responsive to motion-independent spatial information in the model.

Each of the 24 stimuli in the experiment (six endpoint aspect ratios for ascending and six for descending trials in both the no-perturbation and perturbation conditions) was tested 32 times for each of the 4 experimental participants. Therefore, there were 128 trials per stimulus in the simulation. None of these trials were rejected because of either splitting failure (the perception of horizontal and vertical motion signified during the same frame) or coupling failure (motion perception signified for only one of the four motion directions during a frame).

The averaged experimental results and the corresponding quantitative simulations for the no-perturbation and perturbation conditions are presented in Figure 6. In both experiment and simulation, positive hysteresis was observed in the no-perturbation condition; that is, switching was signified at higher aspect ratios for ascending than for descending trials. ${ }^{3}$ In both experiment and simulation, negative (reversed) hysteresis was observed in the perturbation $(\mathrm{BRLC}=0)$ condition; switching was signified at lower aspect ratios for descending than for ascending trials. Calculated values of hysteresis $(H)$ are indicated within each panel of Figure 7.

The negative hysteresis in the perturbation condition was attributable to the effect of adaptation, which would reduce activation primarily for the motion directions that had received above-threshold activation prior to the perturbation (e.g., vertical motion directions for trials with ascending aspect ratios). This was confirmed by the results of additional simulations in which the contribution of adaptation was removed. As anticipated, in this case the negative hysteresis in the perturbation condition was eliminated. That is, switching was signified at the same endpoint aspect ratios for trials with ascending and descending aspect ratios (the hysteresis measure for the simulation increased from $H=-0.14$ to $H=-0.02$ when adaptation was eliminated from the model). For the same reason, $H$ increased from 0.74 to 1.09 in the no-perturbation condition when adaptation was removed, consistent with evidence that adaptation reduces the size of experimentally observed hysteresis (Gepshtein \& Kubovy, 2005; Hock, Kelso, \& Schöner, 1993).

The central hypothesis in this study is that perceptual stability and the occurrence of hysteresis for the motion quartet can result from future-shaping inhibitory interactions. If, for example, vertical motions are activated at above-threshold levels during a frame, the horizontal motion detectors that will be stimulated during the next frame will be inhibited, predisposing the continued perception of vertical motion. This is illustrated in Figure 7 by singletrial simulations with ascending aspect ratios (decreasing stimulus-initiated vertical activation) for pairs of horizontal and vertical motions stimulated during alternating frames. The "zoom" circles magnify the activation levels for downward and upward motions that are not stimulated, but are about to be stimulated during the next frame, when the stimulus-initiated activation of horizontal motion will exceed the stimulus-initiated activation of vertical motion. Whether or not there is a perceptual switch to horizontal motion depends on these subthreshold activation levels.

In the simulated no-perturbation trial (Figure 7A), the subthreshold activation of horizontal motion is lower than the subthreshold activation of vertical motion. This difference in subthreshold activation reflects the future-shaping inhibitory effects of the vertical detectors activated during that frame on the activation of the horizontal detectors that will be stimulated during the next frame. The futureshaped advantage of vertical motion is sufficient to make the continued perception of the vertical motion pattern (and thereby, hysteresis) likely, despite stimulus conditions now favoring the perception of the horizontal motion pattern.

In the simulated perturbation $(B R L C=0)$ trial, the magnification indicates that the subthreshold activation levels of horizontal and vertical motion are the same just before the frame in which the stimulus-initiated activation 


\section{Experimental Results}
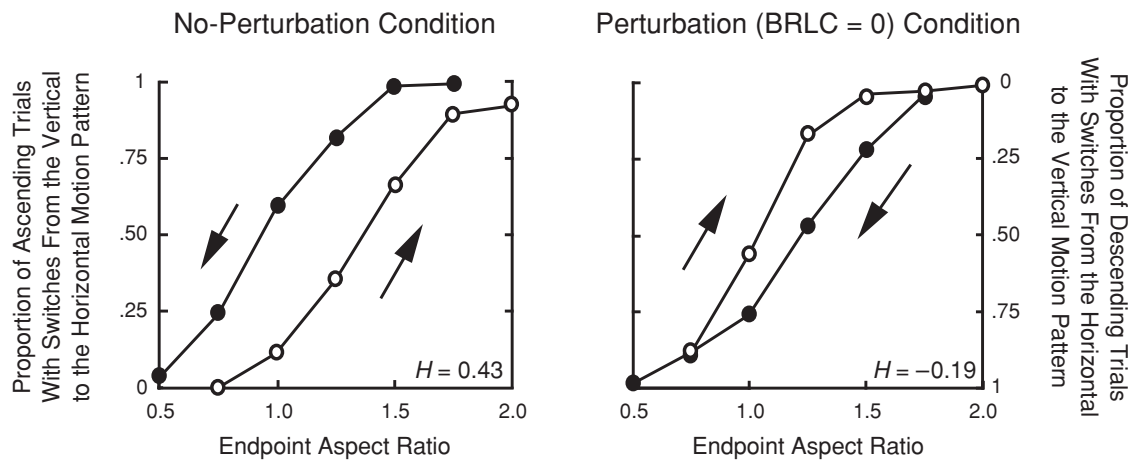

Simulations

No-Perturbation Condition

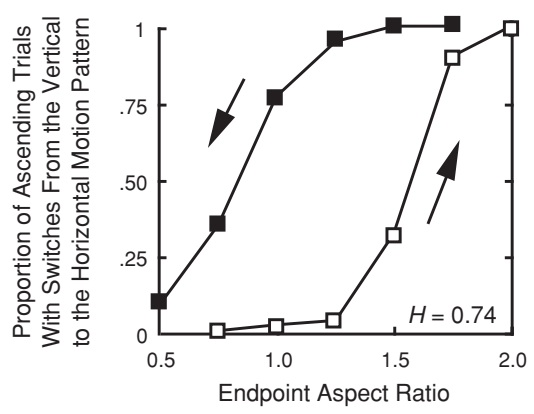

Perturbation $(B R L C=0)$ Condition

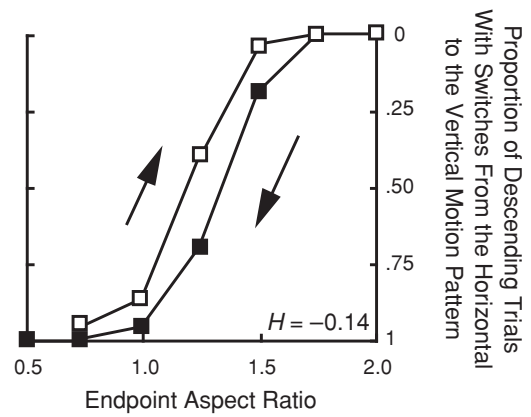

Figure 6. Averaged experimental results for the no-perturbation and perturbation $(B R L C=0)$ conditions of Experiment 1 and their simulations.

of vertical motion will drop below the stimulus-initiated activation of horizontal motion (Figure 7B). This loss of future-shaping, which is attributable to the BRLC $=0$ perturbation briefly eliminating the feedforward of activation from V1 to MT, means that the motion direction that will prevail is determined by the forthcoming stimulus values, which favor the perception of horizontal motion (and thereby, the loss of hysteresis, as indicated in Figure 6).

\section{Experiment 2}

In this experiment, the perturbation increased the BRLC from 0.8 to 2.0 during the next-to-last display cycle. This was implemented in the model by increasing the stimulusinitiated activation of vertical and horizontal motion detectors by 6 during the perturbation. As in Experiment 1, there were 128 trials per stimulus in the simulation. None of these trials were rejected because of either splitting or coupling failure. The averaged experimental results and the corresponding quantitative simulations for the noperturbation and perturbation $(B R L C=2.0)$ conditions are presented in Figure 8. In both experiment and simulation, the size of the hysteresis is larger in the perturbation than the no-perturbation condition.

It is noteworthy that the hysteresis effect for the noperturbation condition was smaller in Experiment 2 than in Experiment 1. This difference, which was observed both empirically and computationally, was attributable to the introduction of an extra pair of frames into the no-perturbation trials of Experiment 2 that repeated the next-to-last aspect ratio of each trial (their purpose was to balance motion adaptation in the perturbation and noperturbation conditions). The extra pair of frames provided additional opportunity for adaptation to reduce the activational advantage of currently perceived motions and for random fluctuations in activation to produce hysteresisreducing perceptual switching (as observed experimentally for motion quartets by Hock et al., 1993).

The difference in the size of the hysteresis between the no-perturbation and perturbation $(\mathrm{BRLC}=2.0)$ conditions again could be accounted for by the activation dependence of inhibitory, future-shaping interactions. This can be seen in Figure 9 for simulations of single trials with decreasing stimulus-initiated vertical activation. Just before the frame during which stimulus-initiated activation was to be smaller for vertical than for horizontal motion, the vertical advantage in subthreshold activation was greater in the perturbation than in the no-perturbation condition. The small increase in the size of the subthreshold difference between vertical and horizontal activation due to the perturbation (i.e., the small increase in future shaping) was consistent with the small increase in hysteresis in both experiment and simulations. 
A

No-Perturbation Condition

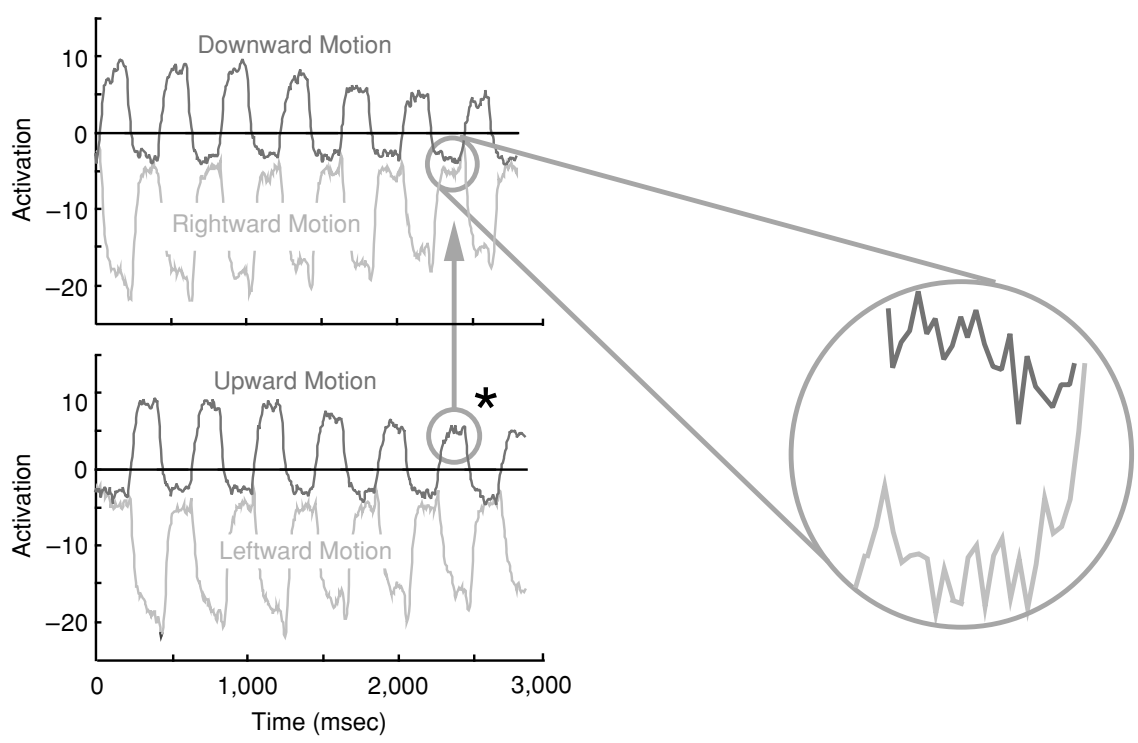

B Perturbation $(B R L C=0)$ Condition

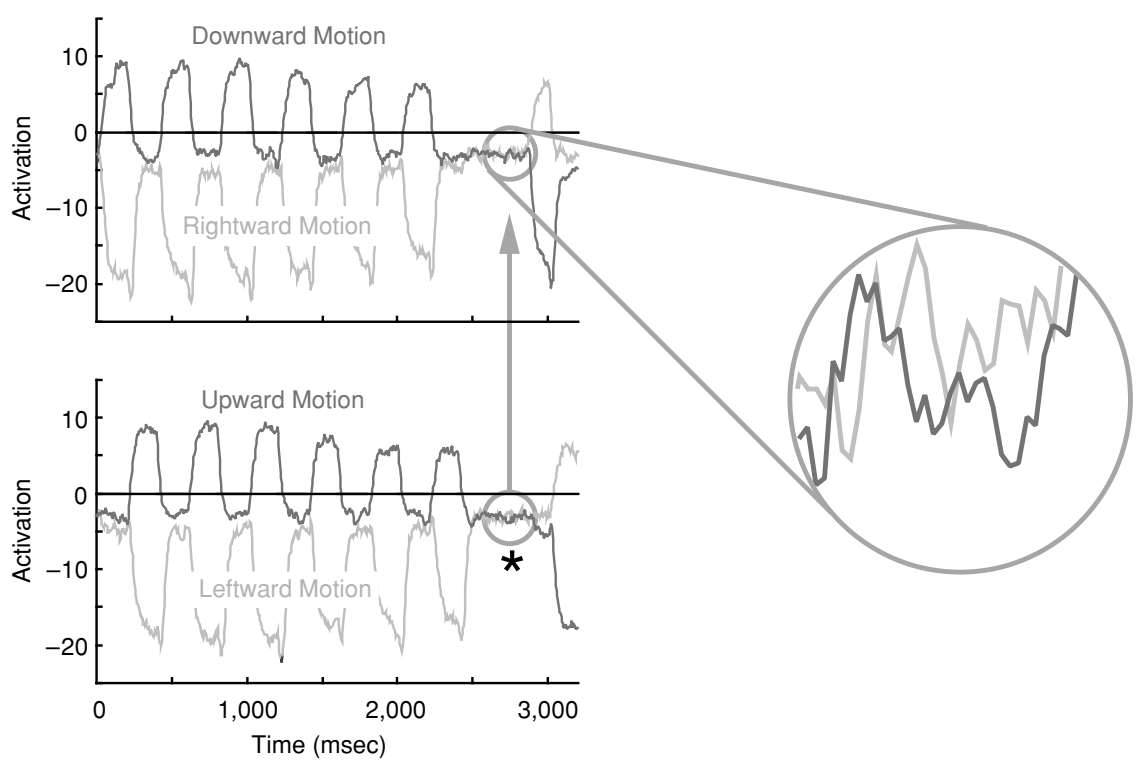

Figure 7. Simulations of single trials with ascending aspect ratios (i.e., decreasing stimulus activation of vertical motion detectors) for the no-perturbation condition (A) and the perturbation $(B R L C=0$ ) condition (B) of Experiment 1. The asterisks denote the stable, fixed-point activation for the simulated detectors, just before the frame during which stimulus-initiated activation values will favor a switch from the perception of a vertical to the perception of a horizontal motion pattern. The "zoom" circles magnify the size of the future-shaping interaction. If the latter is large enough, the perception of vertical motion will continue to be signified. This was the case in the no-perturbation condition, for which hysteresis was obtained, but not in the perturbation (BRLC $=0$ ) condition, for which hysteresis was not obtained (Figure 6).

\section{Experiment 3}

In this experiment, the perturbation decreased the BRLC from 0.8 to 0.4 during the next-to-last display cycle. In order to simulate the distribution of motion-to-nonmotion switches observed in the experiment, the path length de- pendence of stimulus-initiated motion detector activations was reduced during the BRLC perturbation; the range of vertical motion detector activations was changed from a range of $11-17$ in steps of 1 to $9.4-11.2$ in steps of 0.3 . There were 160 trials per stimulus in the simulation of 


\section{Experimental Results}
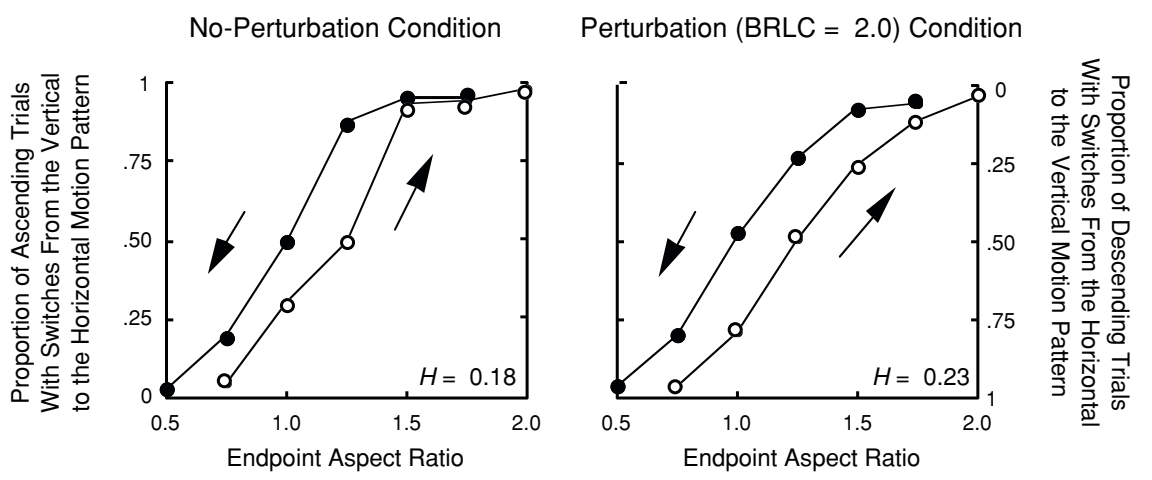

\section{Simulations}
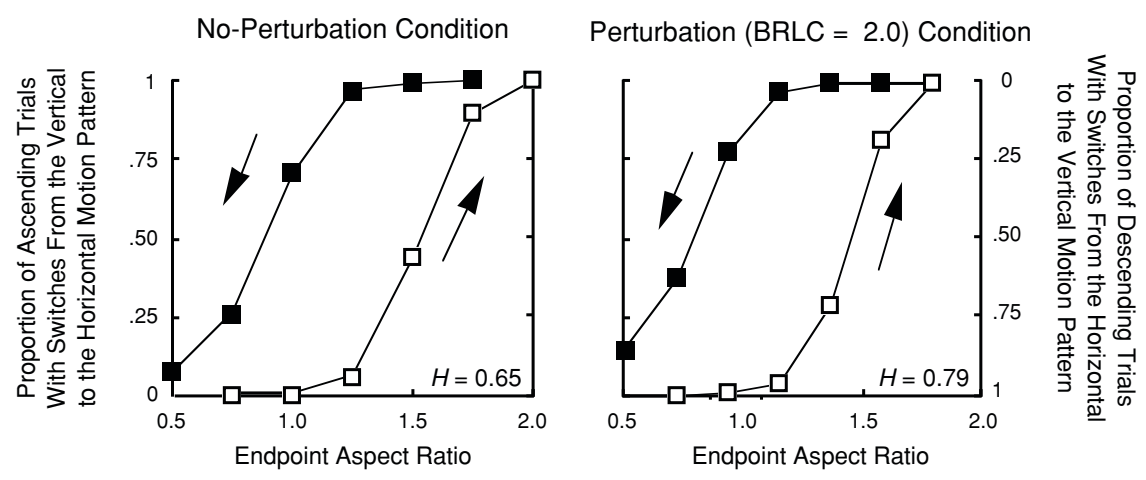

Figure 8. Averaged experimental results for the no-perturbation condition and the perturbation (BRLC $=2.0$ ) condition of Experiment 2 and their simulations.

Experiment 3. None of the trials were discarded because of splitting failure, and fewer than $1.0 \%$ of the perturbation trials were discarded because of coupling failure. For the remaining trials, hysteresis effects were determined separately for trials during which motion versus nonmotion was signified during the perturbation interval.

The frequency with which nonmotion was perceived as a result of the BRLC $=0.4$ perturbation was simulated (Figure 10), as were the consequences of the motion/ nonmotion bistability for the size of pattern-level hysteresis in the perturbation condition (Figure 11). Hysteresis was obtained in both experiment and simulation when motion was perceived during the perturbation, and reverse hysteresis was obtained in both experiment and simulation when nonmotion was perceived during the perturbation.

The simulations of single descending trials in Figure 12 again focus on the future-shaping inhibition of horizontal motion detectors by currently activated vertical motion detectors. Of interest once again is the size of the vertical/horizontal difference in subthreshold activation just before the frame during which the stimulus-initiated activation of horizontal detectors exceeds the decreasing stimulus-initiated activation of vertical detectors (favoring a perceptual switch from a vertical to a horizontal motion pattern). For trials during which motion is perceived throughout (Figure 12A), the vertical/horizontal difference in subthreshold activation was sufficiently large to overcome changes in stimulus-initiated activation favoring the perception of the horizontal motion pattern, so hysteresis was signified (as indicated in Figure 11). However, for trials during which nonmotion was perceived during the perturbation (Figure 12B), the vertical/horizontal difference in subthreshold activation was eliminated during the perturbation (as in the simulation for the BRLC $=0$ perturbation in Experiment 1), so changes in stimulusinitiated activation resulted in a switch to the perception of the horizontal motion pattern immediately after motion resumed, and the elimination of hysteresis was signified (as indicated in Figure 11).

\section{Overview of Results}

The results of the simulations described above demonstrate the computational plausibility of a dynamical theory of multilevel pattern formation. The model quantitatively simulated the relative size of the hysteresis effects for all three experiments reported by Hock and Ploeger (2006), and simulations of individual trials confirmed that future-shaping inhibitory interactions are the basis for the effects of local-level BRLC perturbations on the relative size of pattern-level hysteresis. These interactions pro- 


\section{A No-Perturbation Condition}

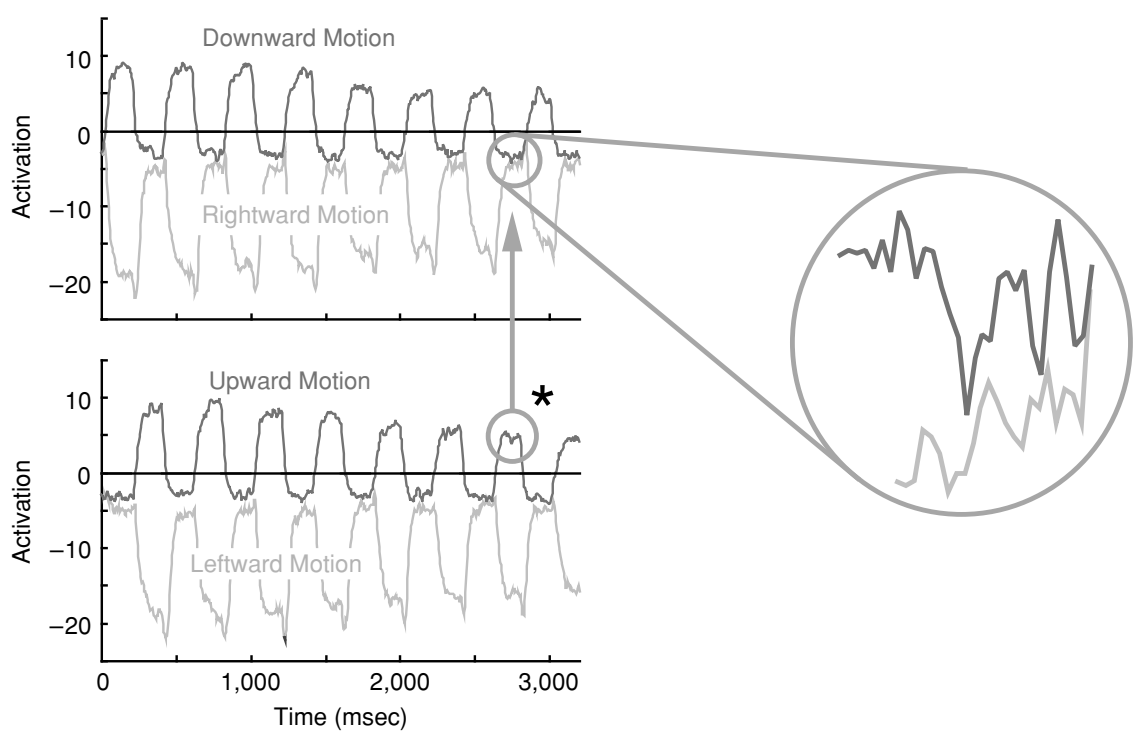

B Perturbation $(B R L C=2.0)$ Condition

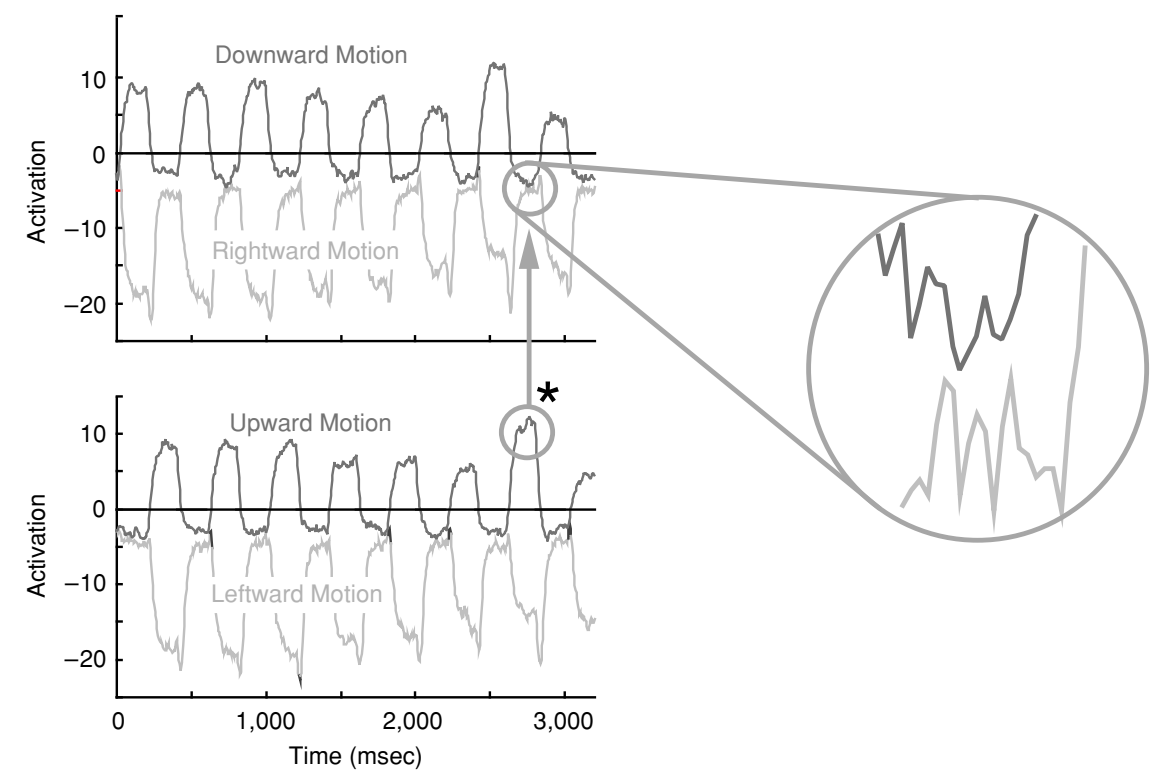

Figure 9. Simulations of single trials with ascending aspect ratios (i.e., decreasing stimulus activation of vertical motion detectors) for the no-perturbation condition (A) and the perturbation ( $B R L C=2.0$ ) condition $(B)$ of Experiment 2. The asterisks denote the stable, fixed-point activation for the simulated detectors, just before the frame during which stimulus-initiated activation values will favor a switch from the perception of a vertical to the perception of a horizontal motion pattern. The "zoom" circles magnify the size of the future-shaping interaction. If the latter is large enough, the perception of vertical motion will continue to be signified. This was the case in the no-perturbation condition, in which hysteresis was obtained, and even more so in the perturbation $(B R L C=2.0)$ condition, in which hysteresis increased (Figure 8).

moted hysteresis by introducing a subthreshold advantage in activation that favored the continued perception of the currently perceived pattern. The activation dependence of the future-shaping interactions is illustrated in Figure 13, which re-presents the nonlinear interaction function from
Figure 5B, but now with the superposition of the stable, fixed points from the three experiments. The latter are the activations for the motion (or nonmotion) perceived during the perturbation (indicated by asterisks in Figures 7, 9, and 12), just before stimulus-initiated activation is going 

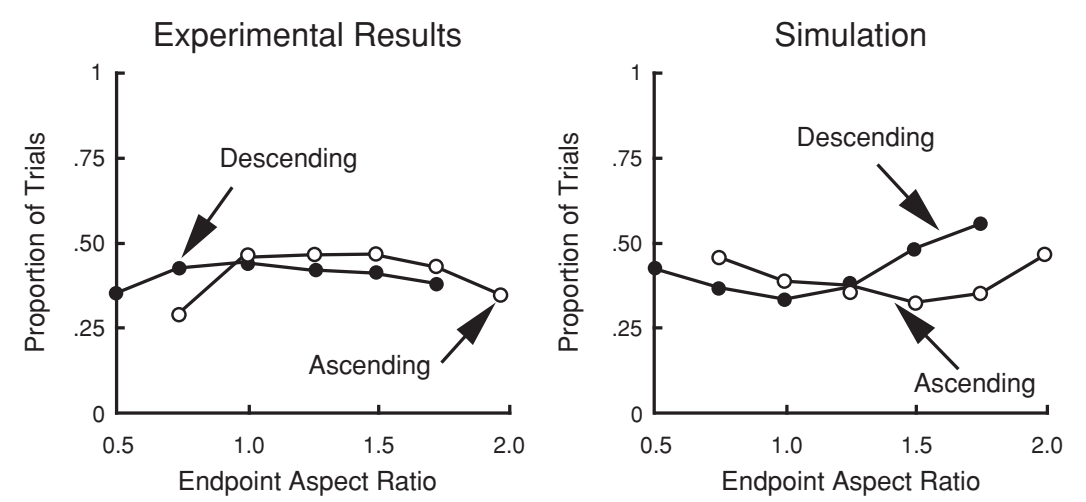

Figure 10. The proportion of trials in the perturbation $(B R L C=0.4)$ condition of Experiment 3 and its simulation in which nonmotion was perceived. (The experimental results are averaged over the 4 participants.)

to favor a switch in the perceived pattern. When the fixedpoint values for the various experimental conditions are arrayed along the "activation axis" of the interaction function, it can be seen that they are in ordinal correspondence with the size of the hysteresis effect $(H)$ for each condition. This is consistent with the effect of future-shaping inhibitory interactions on soon-to-be stimulated motions (and thereby, on the size of hysteresis) depending on the activation level of the detectors initiating the inhibition.

As indicated earlier, the same parameters were used in the simulations for all of Hock and Ploeger's (2006) results. Even though there was no attempt to simulate other experimental results in selecting these parameter values, it turned out that the simulations also accounted for earlier experimental findings by Hock et al. (1993). The latter findings included the effect of adaptation in reducing hysteresis (simulations for Experiment 1) and the reduction in hysteresis observed when frames with the same aspect ratio were repeated within ascending and descending trials (the comparison of hysteresis in the no-perturbation simulations for Experiments 1 and 2).

\section{DISCUSSION}

The classical conceptualization of hierarchical pattern formation assumes that small units are formed, then larger ones built from the smaller ones, and so on, at successively higher levels of description. This idea, which assumes that processing is complete (the unit is formed) at each level before the next-larger unit is built, is not neurally plausible. Because of myelination differences, activation passes between levels in the visual system much more rapidly than it passes between neurons within the same level (Gilbert, 1992), too rapidly for processing to be complete at one level before it is initiated at the next (e.g., neural transmission from V1 to MT takes 1-2 msec; Movshon \& Newsome, 1996). The neat "building-block" picture is made obsolete by such rapid interlevel transmission, but a much more dynamic picture emerges as a result. That is, processing at a higher level can be influenced almost immediately by feedforward processing decisions that are just beginning to emerge at a lower level, and there is ample opportunity for processing at the higher level to influence lower-level processing through feedback.

Hock and Ploeger's (2006) psychophysical results and the computational model developed in this article have focused on the feedforward aspect of activation flow between different levels of processing. In line with previous research, we assumed that local motion/nonmotion perceptual decisions are made in area V1 and patternlevel perceptual decisions are made in area MT. In both the experiments and simulations, local motion activation was manipulated through the introduction of perturbations in luminance that briefly changed stimulus-initiated motion detector activation. Our findings showed that perturbations' effects on pattern-level hysteresis depend on whether the stimulus-initiated activation is decreased (Experiment 1) or increased (Experiment 2). The futureshaping interactions that are crucial for pattern stability (and hysteresis) are activation dependent, so perturbations that reduce feedforward activation decrease pattern-level hysteresis by reducing the effectiveness of future-shaping interactions. Conversely, luminance perturbations that boost feedforward activation increase pattern-level hysteresis by increasing the extent to which future-shaping interactions predispose the continued perception of the currently perceived motion pattern. Finally, we showed that the effects of local luminance perturbations on patternlevel hysteresis are due to differences in feedforward activation rather than differences in stimulation. When a perturbation moderately reduced the stimulus-initiated activation of motion detectors, there was local bistability for the same stimulus, and either motion or nonmotion was perceived during the perturbation. For the same change in stimulus-initiated detector activation, the size of the pattern-level hysteresis depended on the outcome of this local motion/nonmotion decision (Experiment 3).

Future-shaping inhibition of competing motion directions stabilizes percepts for stimuli like the motion quartet because the same directionally selective motion detector is not stimulated twice in succession; instead, during an intervening period of time, movement in the opposite di- 


\section{Experimental Results}

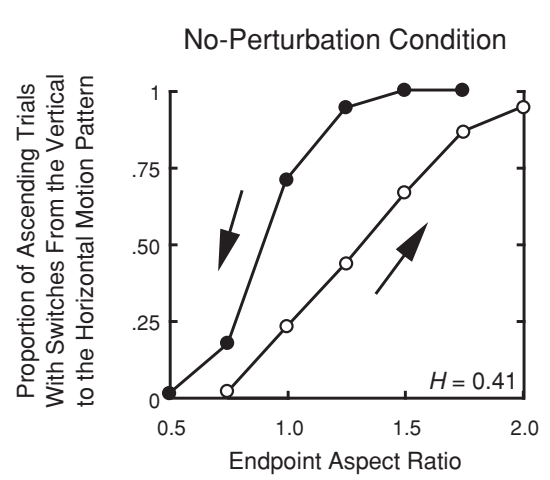

No-Perturbation Condition
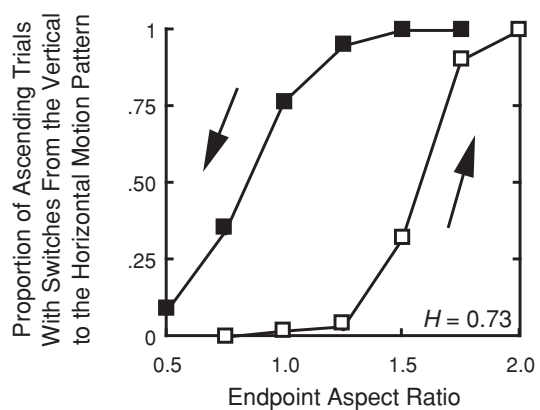

Perturbation $(B R L C=0.4)$

Condition: Motion Is

Perceived for Entire Trial

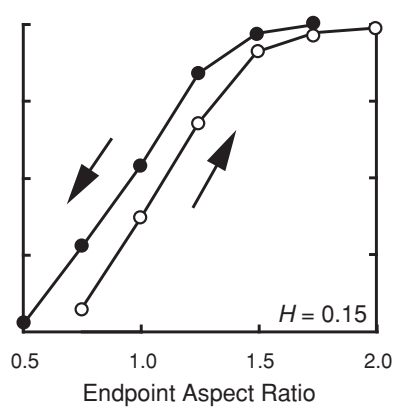

Simulations

Perturbation $(B R L C=0.4)$

Condition: Motion Is

Perceived for Entire Trial

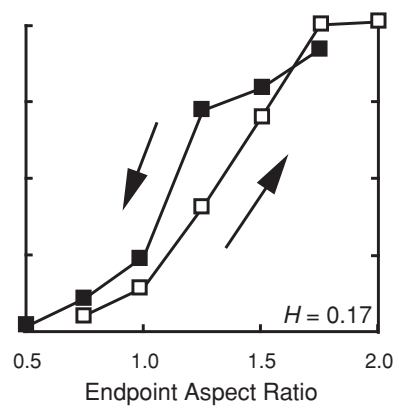

Perturbation $(\mathrm{BRLC}=0.4)$

Condition: Nonmotion

Is Perceived

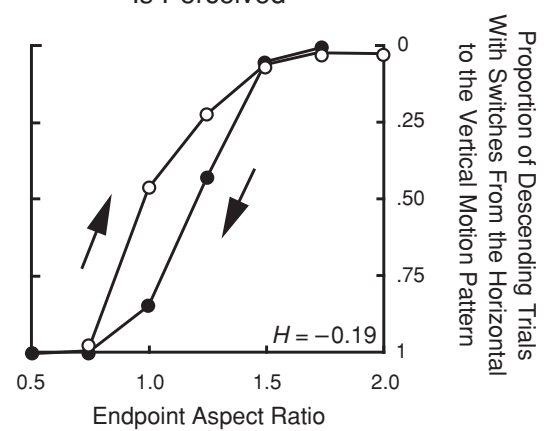

Perturbation $(B R L C=0.4)$
Condition: Nonmotion

Is Perceived

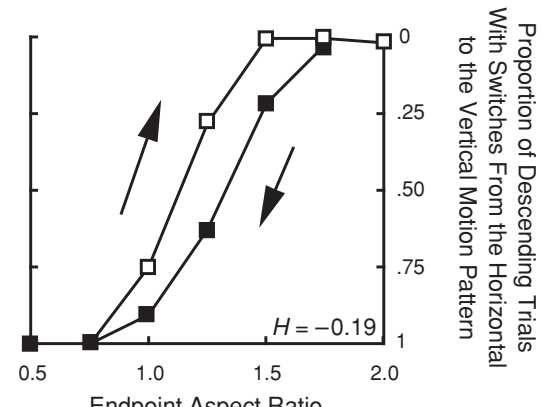

Figure 11. Averaged experimental results for the no-perturbation and perturbation (BRLC $=0.4$ ) conditions of Experiment 3 and their simulations. The results for the perturbation condition are presented separately for trials in which motion was perceived/signified throughout the trial and for trials in which nonmotion was perceived/signified during the trial.

rection is stimulated along the same path. Future shaping through the persistence of activation, though it might be considered a more intuitive basis for hysteresis, cannot bridge the interval between successive stimulations of the same detector for the motion quartet. This does not, of course, rule out the possibility that the persistence of activation stabilizes perceived motion in other circumstances (e.g., when the same motion direction is continually restimulated by a relatively large textured surface moving across the visual field). Future shaping also can result from activation-facilitating excitatory interactions. This is indicated by evidence for the priming of motion direction in Anstis and Ramachandran's (1987) study of motion inertia, by the continued perception of motion in the same direction for directionally ambiguous apparent motion stimuli (Hock \& Balz, 1994), and by the long-term stabilization of a percept resulting from the periodic introduction of blank intervals (Leopold, Wilke, Maier, \& Logothetis, 2002; Orbach, Ehrlich, \& Heath, 1963). Relevant to the latter finding is the simplifying assumption in our simulations that the stimulus activation of motion detectors con- tinues over the full 200-msec duration of each frame. If frame duration were substantially longer than the duration of motion detector activation, future-shaping inhibitory interactions would likely become a less effective basis for hysteresis, and slow-time-scale future-shaping excitatory interactions would potentially become a more important contributor to pattern stability and hysteresis.

There have been many earlier dynamical models in which inhibition among motion detectors with different directional selectivity has been the basis for pattern formation (Bartsch \& van Hemmen, 1997; Carmesin \& Arndt, 1996; Hock et al., 2003; Kawamoto \& Anderson, 1985; Williams, Phillips, \& Sekuler, 1986; Wilson \& Kim, 1994), and there have also been dynamical multilevel models entailing motion processing in both areas V1 and MT (Chey, Grossberg, \& Mingolla, 1997; Grossberg, Mingolla, \& Viswanathan, 2001). ${ }^{4}$ However, other than Hock et al.'s (2003) model, none of these have identified future-shaping interactions as the basis for the stability of perceived patterns. The present model carries this concept a step farther, emphasizing the activation dependence of future-shaping interactions. 

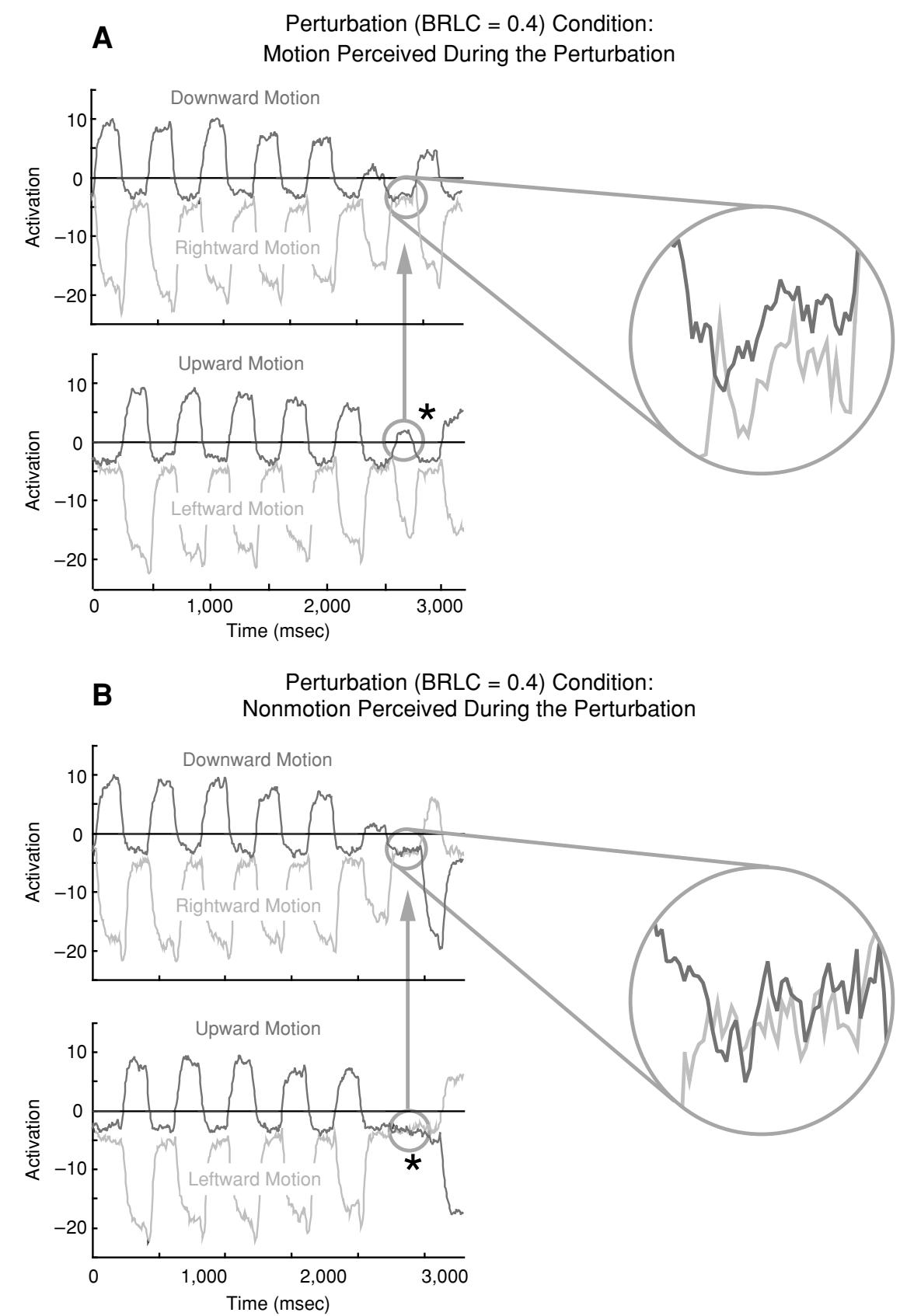

Figure 12. Simulations of single trials with ascending aspect ratios (i.e., decreasing stimulus activation of vertical motion detectors) for the perturbation $(B R L C=0.4)$ condition of Experiment 3, presented separately for trials in which motion was perceived during the perturbation (A) and trials in which nonmotion was perceived during the perturbation (B). The asterisks denote the stable, fixed-point activation for the simulated detectors, just before the frame during which stimulus-initiated activation values will favor a switch from the perception of a vertical to the perception of a horizontal motion pattern. The "zoom" circles magnify the size of the future-shaping interaction. If the latter is large enough, the perception of vertical motion will continue to be signified. This was the case when motion was perceived during the perturbation (and hysteresis was obtained), but not when nonmotion was perceived (and hysteresis was not obtained; Figure 11).

In addition, previous models that have been concerned with the relationship between motion detection and the detection of motion-independent spatial information have focused on the integration of motion and form (Baloch \&
Grossberg, 1997; Francis \& Grossberg, 1996). Heretofore, however, no models have explicitly introduced competition between the detectors that are the basis of form and motion perception and shown as well that the competitive interac- 


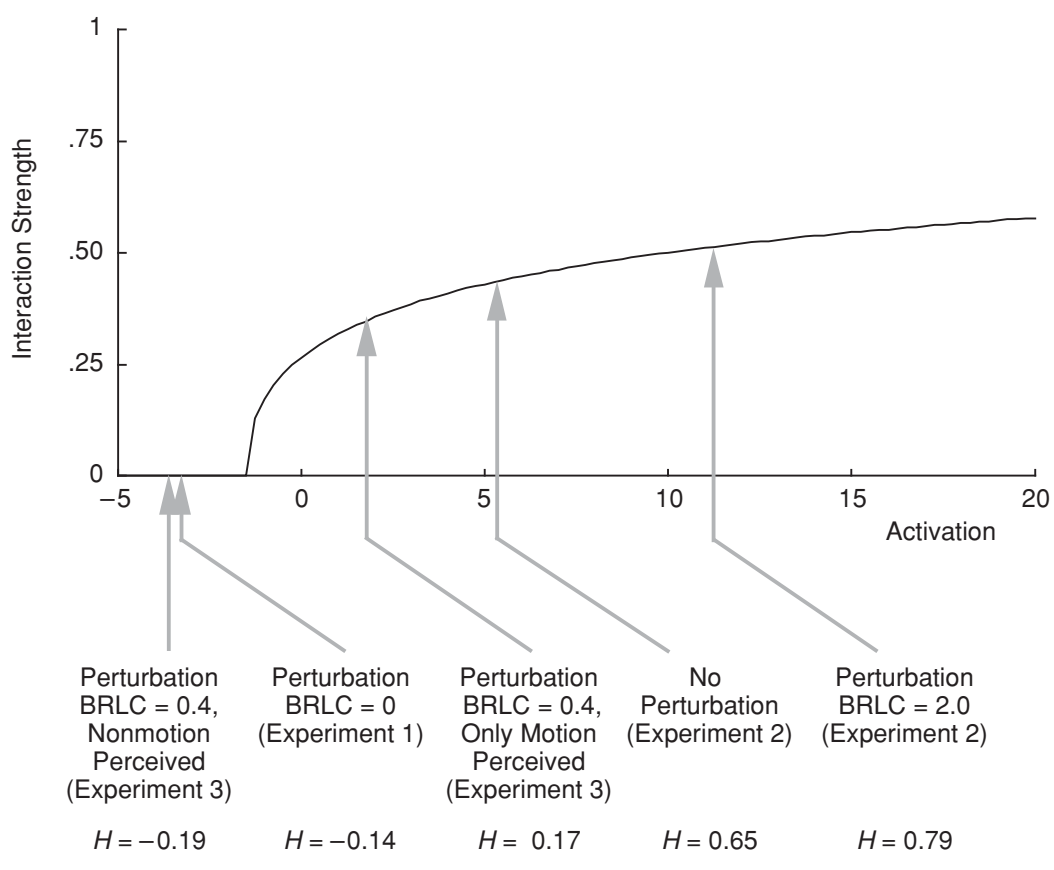

Figure 13. Nonlinear function representing inhibitory interactions among motion detectors with orthogonal directional selectivity in area MT (as in Figure 5C).Arrayed along the activation axis are the stable fixed-point activation values for the perceived motions signified in the simulations of all three experiments, just before the frame during which changes in the stimulus favored the alternative motions. These are the activation values marked by asterisks in the single-trial simulations presented in Figures 7,9, and 12. (Motion was not perceived during perturbations in which activation was subthreshold.) Presented with each fixed-point activation value is the hysteresis effect $(H)$ for the indicated experimental condition, as determined by the quantitative simulations presented in Figures 6, 8, and 11.

tions that determine whether or not motion is perceived can affect the strength of the motion detector interactions that are the basis of the stability of perceived motion patterns.

In conclusion, the results of a number of experiments by Hock and Ploeger (2006) and Hock et al. (1993) have been simulated by a computational model in which the level of feedforward activation between local (area V1) and pattern (area MT) levels of processing affects the size of pattern-level hysteresis. This emphasis on the feedforward flow of activation does not preclude the role of feedback from MT to V1, nor of feedback from other brain areas (e.g., from MST to MT). Evidence for the former comes from Shim and Cavanagh (2004), who have shown that the perceived alignment of flanking lines on opposite sides of a motion quartet (presumably based on V1 detectors responsive to motion-independent spatial information) is affected by whether horizontal or vertical motion is perceived for the quartet (presumably determined by inhibitory detector interactions in MT). Evidence for feedback from MST to MT comes from a study currently underway in our laboratory. We have found that when four quartets are presented in a spatial configuration that results in the perception of rotation, the perceived motions for two of the quartets are in directions that are clearly inconsistent with their aspect ratio. The perception of horizontal versus vertical motion patterns for each motion quartet depends on pattern-forming inhibitory interactions among directionally selective MT motion detectors (see, e.g., Thiele et al., 2000), so the modification of these interactions in the four-quartet configuration implicates feedback to MT from rotation-selective neurons in MSTd (Tanaka \& Saito, 1989). Experimental findings like these will be the basis for the expansion of the present model to test the plausibility of a dynamical theory of multilevel pattern formation that includes feedback as well as the feedforward flow of activation.

\section{REFERENCES}

Anstis, S., Giaschi, D., \& Cogan, A. I. (1985). Adaptation to apparent motion. Vision Research, 25, 1051-1062.

ANSTIS, S., \& RAMACHANDRAN, V. S. (1987). Visual inertia in apparent motion. Vision Research, 27, 755-764.

Baloch, A. A., \& Grossberg, S. (1997). A neural model of high-level motion processing: Line motion and formotion dynamics. Vision Research, 37, 3037-3059.

Banta, A. R., \& Breitmeyer, B. G. (1985). Stationary patterns suppress the perception of stroboscopic motion. Vision Research, 25, 1501-1505.

Bartsch, A., \& van Hemmen, J. L. (1997). A neural model of stroboscopic alternative motion. Network, 8, 107-126. 
Breitmeyer, B., Love, R., \& Wepman, B. (1974). Contour suppression during stroboscopic motion and metacontrast. Vision Research, 14, 1451-1456.

Carandini, M., \& Ferster, D. (1997). A tonic hyperpolarization underlying contrast adaptation in cat visual cortex. Science, 276, 949-952.

Carmesin, H. O., \& ARndT, S. (1996). A neural network model for stroboscopic alternative motion. Biological Cybernetics, 75, 239-251.

Chaudhuri, A., \& Glaser, D. A. (1991). Metastable motion anisotropy. Visual Neuroscience, 7, 397-407.

Chey, J., Grossberg, S., \& Mingolla, E. (1997). Neural dynamics of motion grouping: From aperture ambiguity to object speed and direction. Journal of the Optical Society of America A, 14, 2570-2594.

Eastman, K. E., \& Hock, H. S. (1999). Bistability in the perception of motion and stationarity: Effects of temporal asymmetry. Perception \& Psychophysics, 61, 1055-1065.

Francis, G., \& Grossberg, S. (1996). Cortical dynamics of form and motion integration: Persistence, apparent motion, and illusory contours. Vision Research, 36, 149-173.

GARDINER, C. W. (1983). Handbook of stochastic methods for physics, chemistry, and the natural sciences. Berlin: Springer.

Gepshtein, S., \& Kubovy, M. (2005). Stability and change in perception: Spatial organization in temporal context. Experimental Brain Research, 160, 487-495.

Giaschi, D., Douglas, R., Marlin, S., \& Cynader, M. (1993). The time course of direction-selective adaptation in simple and complex cells in cat striate cortex. Journal of Neurophysiology, 70, 2024 2034.

Gilbert, C. D. (1992). Horizontal integration and cortical dynamics. Neuron, 9, 1-20.

Gilroy, L. A., Hock, H. S., \& Ploeger, A. (2001). Differential activation solution to the motion correspondence problem. Perception \& Psychophysics, 63, 847-861.

GrossberG, S. (1980). Biological competition: Decision rules, pattern formation, and oscillations. Proceedings of the National Academy of Sciences, 77, 2338-2342.

Grossberg, S., Mingolla, E., \& Viswanathan, L. (2001). Neural dynamics of motion integration and segmentation within and across apertures. Vision Research, 41, 2521-2553.

Heeger, D. J., Boynton, G. M., Demb, J. B., Seidemann, E., \& NewSOME, W. T. (1999). Motion opponency in visual cortex. Journal of Neuroscience, 19, 7162-7174.

Hock, H. S., \& BALz, G. W. (1994). Spatial scale dependent in-phase and anti-phase directional biases in the perception of self-organized motion patterns. Vision Research, 34, 1843-1861.

Hock, H. S., Kelso, J. A. S., \& Schöner, G. (1993). Bistability and hysteresis in the organization of apparent motion patterns. Journal of Experimental Psychology: Human Perception \& Performance, 19, 63-80.

Hock, H. S., Kogan, K., \& Espinoza, J. K. (1997). Dynamic, statedependent thresholds for the perception of single-element apparent motion: Bistability from local cooperativity. Perception \& Psychophysics, 59, 1077-1088.

Hock, H. S., Nichols, D. F., \& EspinozA, J. [K.] (2004). When motion is not perceived: Evidence from adaptation and dynamical stability. Spatial Vision, 17, 235-248.

Hock, H. S., \& Ploeger, A. (2006). Linking dynamical perceptual decisions at different levels of description in motion pattern formation: Psychophysics. Perception \& Psychophysics, 68, 505-514.

Hock, H. S., Schöner, G., \& GIESE, M. (2003). The dynamical foundations of motion pattern formation: Stability, selective adaptation, and perceptual continuity. Perception \& Psychophysics, 65, 429-457.

Hock, H. S., Schöner, G., \& Hochstein, S. (1996). Perceptual stability and the selective adaptation of perceived and unperceived motion. Vision Research, 36, 3311-3323.

Huk, A. C., Ress, D., \& Heeger, D. J. (2001). Neuronal basis of the motion aftereffect reconsidered. Neuron, 32, 161-172.

Kawamoto, A. H., \& Anderson, J. A. (1985). A neural network model of multistable perception. Acta Psychologica, 59, 35-65.

Kloeden, P. E., \& Platen, E. (1999). Numerical solution of stochastic differential equations. Berlin: Springer.

KoHn, A., \& Movshon, J. A. (2003). Neuronal adaptation to visual motion in Area MT of the macaque. Neuron, 39, 681-691.
Kruse, P., Stadler, M., \& Wehner, T. (1986). Direction and frequency specific processing in the perception of long-range apparent movement. Vision Research, 26, 327-335.

Kruse, W., Dannenberg, S., Kleiser, R., \& Hoffmann, K.-P. (2002). Temporal relation of population activity in visual areas MT/MST and in primary motor cortex during visually guided tracking movements. Cerebral Cortex, 12, 466-476.

KULIKowsKi, J. J. (1978). Spatial resolution for the detection of pattern and movement (real and apparent). Vision Research, 18, 237-238.

Leopold, D. A., Wilke, M., Maier, A., \& Logothetis, N. K. (2002). Stable perception of visually ambiguous patterns. Nature Neuroscience, 5, 605-609.

Levinson, E., \& Sekuler, R. (1975). Inhibition and disinhibition of direction-specific mechanisms in human vision. Nature, 254, 692-694.

Maunsell, J. H., \& VAn Essen, D. C. (1983). Functional properties of neurons in middle temporal visual area of the macaque monkey: I. Selectivity for stimulus direction, speed, and orientation. Journal of Neurophysiology, 49, 1127-1147.

Movshon, J. A., \& Newsome, W. T. (1996). Visual response properties of striate cortical neurons projecting to area MT in macaque monkeys. Journal of Neuroscience, 16, 7733-7741.

Movshon, J. A., Thompson, I. D., \& Tolhurst, D. J. (1978). Receptive field organization of complex cells in the cat's striate cortex. Journal of Physiology, 283, 79-99.

NaKa, K. I., \& Rushton, W. A. (1966). S-potentials from luminosity units in the retina of fish (Cyprinidae). Journal of Physiology, 185, 587-599.

Orbach, J., Ehrlich, D., \& Heath, H. A. (1963). Reversibility of the Necker cube: I. An examination of the concept of "satiation of orientation." Perceptual \& Motor Skills, 17, 439-458.

Ploeger, A., van der Maas, H. L. J., \& Hartelman, P. A. I. (2002). Stochastic catastrophe analysis of switches in the perception of apparent motion. Psychonomic Bulletin \& Review, 9, 26-42.

Priebe, N. J., Churchland, M. M., \& Lisberger, S. G. (2002). Constraints on the source of short-term motion adaptation in macaque area MT: I. The role of input and intrinsic mechanisms. Journal of Neurophysiology, 88, 354-369.

Recanzone, G. H., Wurtz, R. H., \& Schwarz, U. (1997). Responses of MT and MST neurons to one and two moving objects in the receptive field. Journal of Neurophysiology, 78, 2904-2915.

Sanchez-Vives, M. V., NowAK, L. G., \& McCormick, D. A. (2000). Membrane mechanisms underlying contrast adaptation in cat area 17 in vivo. Journal of Neuroscience, 20, 4267-4285.

SCHÖNER, G., \& Hock, H. S. (1995). Concepts for a dynamical theory of perceptual organization: An example from apparent movement. In P. Kruse \& M. Stadler (Eds.), Ambiguity in mind and nature (pp. 275310). Berlin: Springer.

Shim, W. M., \& CaVAnagh, P. (2004). The motion-induced position shift depends on the perceived direction of bistable quartet motion. Vision Research, 44, 2393-2401.

Snowden, R. J., Treue, S., Erickson, R. G., \& Andersen, R. A. (1991). The response of area MT and V1 neurons to transparent motion. Journal of Neuroscience, 11, 2768-2785.

Sterzer, P., Eger, E., \& Kleinschmidt, A. (2003). Responses of extrastriate cortex to switching perception of ambiguous visual motion stimuli. NeuroReport, 14, 2337-2341.

TANAKA, K., \& SAITO, H.-A. (1989). Analysis of motion of the visual field by direction, expansion/contraction, and rotation cells clustered in the dorsal part of the medial superior temporal area of the macaque monkey. Journal of Neurophysiology, 62, 643-656.

Thiele, A., Dobkins, K. R., \& Albright, T. D. (2000). Neural correlates of contrast detection at threshold. Neuron, 26, 715-724.

Tolias, A. S., Smirnakis, S. M., Augath, M. A., Trinath, T., \& LogoTHETIS, N. K. (2001). Motion processing in the macaque: Revisited with functional magnetic resonance imaging. Journal of Neuroscience, 21, 8594-8601.

VAutin, R. G., \& Berkley, M. A. (1977). Responses of single cells in cat visual cortex to prolonged stimulus movement: Neural correlates of visual aftereffects. Journal of Neurophysiology, 40, 1051-1065.

Williams, D., Phillips, G., \& Sekuler, R. (1986). Hysteresis in the perception of motion direction as evidence for neural cooperativity. Nature, 324, 253-255. 
WILSON, H. R. (1998). Spikes, decisions, and actions: The dynamical foundations of neuroscience. Oxford: Oxford University Press.

Wilson, H. R., \& KIM, J. (1994). A model for motion coherence and transparency. Visual Neuroscience, 11, 1205-1220.

\section{NOTES}

1. There is a well-known bias to perceive vertical motion when the horizontal and vertical interelement distances of the motion quartet are equal (i.e., when the aspect ratio is 1.0; Chaudhuri \& Glaser, 1991; P. Kruse, Stadler, \& Wehner, 1986). This was attributed by Gilroy et al. (2001) to greater sensitivity to vertical than to horizontal motion for the same interelement distance. Equal horizontal and vertical activation values in the simulations therefore corresponded to an aspect ratio of the motion quartet that was somewhat greater than 1.0.

2. In Hock and Ploeger's (2006) experiments, the effects of the luminance perturbations were distributed over frames leading into and out of the pair of perturbation frames. This was not fully captured in the modeling, which limited the perturbation to two frames. As a result, the effect of the perturbations may have been somewhat underestimated in the simulations.

3. Hysteresis effects in the no-perturbation condition were larger in the simulation than in the actual experiment. This difference might have been reduced if the model included slower-time-scale adaptation effects accumulating across multiple trials.

4. Dynamical modeling for the motion quartet has also been based on catastrophe analysis (Ploeger, van der Maas, \& Hartelman, 2002).

\section{APPENDIX}

The two-level dynamical model for the motion quartet (Figure 1) is based on 20 coupled differential equations representing the activational states of four local (area V1) detectors responsive to motion-independent spatial information, eight local (area V1) detectors responsive to motion-specifying stimulus information, and eight pattern-level (area MT) motion detectors that receive feedforward activation from area V1 motion detectors with corresponding directional selectivity. Each local (V1) detector is represented by an activation variable, $u_{i}^{\mathrm{V} 1}$, where $i=\mathrm{Br}, \mathrm{Bl}, \mathrm{Tr}, \mathrm{Tl}, \mathrm{Ru}, \mathrm{Rd}, \mathrm{Lu}, \mathrm{Ld}, \mathrm{Bp}, \mathrm{Tp}, \mathrm{Rp}$, or Lp. The first eight values of $i$ denote motion detectors, and the last four values denote oriented, motion-independent detectors that respond to the elements on each side of the quartet. The MT motion detectors are represented by an activation variable, $u_{i}^{\mathrm{MT}}$, where $i=\mathrm{Br}, \mathrm{Bl}, \mathrm{Tr}$, $\mathrm{Tl}, \mathrm{Ru}, \mathrm{Rd}, \mathrm{Lu}$, or $\mathrm{Ld}$. For each activation variable $u_{i}$, there is an adaptation variable, $v_{i}$, whose activation will increase by an amount proportional to the current activation level of $u_{i}$. Its value is subtracted from the activation of the corresponding activation variable.

The dynamics of the local-level (V1) detectors are described below. The rate of change of the activation variable $u_{i}^{\mathrm{V} 1}$ is determined by the time scale of activation change, $\tau$, the no-stimulus resting level, $h^{\mathrm{V} 1}$, the stimulusinitiated activation, $S_{i}(t)$, the adaptation level, $v_{i}^{\mathrm{V} 1}$, additive Gaussian white noise, $\xi(t)$, with strength $q$, as well as by interactions from other local-level detectors. Inhibitory coupling with other activational variables is denoted by $\sigma^{\mathrm{V} 1}\left(u_{j}^{\mathrm{V} 1}\right)$, with $\omega_{\mathrm{V} 1}$ denoting the maximum strength of the coupling.

The stochastic differential equations (Gardiner, 1983) for the V1 detectors responsive to motion-independent spatial information are

$$
\begin{aligned}
\tau \frac{d u_{\mathrm{Bp}}^{\mathrm{V} 1}}{d t}= & -u_{\mathrm{Bp}}^{\mathrm{V} 1}+h^{\mathrm{V} 1}+S_{\mathrm{Bp}}(t)-v_{\mathrm{Bp}}^{\mathrm{V} 1}-\omega_{\mathrm{V} 1} \sigma^{\mathrm{V} 1}\left(u_{\mathrm{Br}}^{\mathrm{V} 1}\right)-\omega_{\mathrm{V} 1} \sigma^{\mathrm{V} 1}\left(u_{\mathrm{B} 1}^{\mathrm{V} 1}\right) \\
& -\omega_{\mathrm{V} 1} \sigma^{\mathrm{V} 1}\left(u_{\mathrm{Ru}}^{\mathrm{V} 1}\right)-\omega_{\mathrm{V} 1} \sigma^{\mathrm{V} 1}\left(u_{\mathrm{Rd}}^{\mathrm{V} 1}\right)-\omega_{\mathrm{V} 1} \sigma^{\mathrm{V} 1}\left(u_{\mathrm{Lu}}^{\mathrm{V} 1}\right)-\omega_{\mathrm{V} 1} \sigma^{\mathrm{V} 1}\left(u_{\mathrm{Ld}}^{\mathrm{V} 1}\right)+q \xi_{\mathrm{Bp}}^{\mathrm{V} 1}(t), \\
\tau \frac{d u_{\mathrm{Tp}}^{\mathrm{V} 1}}{d t}= & -u_{\mathrm{Tp}}^{\mathrm{V} 1}+h^{\mathrm{V} 1}+S_{\mathrm{Tp}}(t)-v_{\mathrm{Tp}}^{\mathrm{V} 1}-\omega_{\mathrm{V} 1} \sigma^{\mathrm{V} 1}\left(u_{\mathrm{Tr}}^{\mathrm{V} 1}\right)-\omega_{\mathrm{V} 1} \sigma^{\mathrm{V} 1}\left(u_{\mathrm{T} 1}^{\mathrm{V} 1}\right) \\
& -\omega_{\mathrm{V} 1} \sigma^{\mathrm{V} 1}\left(u_{\mathrm{Ru}}^{\mathrm{V} 1}\right)-\omega_{\mathrm{V} 1} \sigma^{\mathrm{V} 1}\left(u_{\mathrm{Rd}}^{\mathrm{V} 1}\right)-\omega_{\mathrm{V} 1} \sigma^{\mathrm{V} 1}\left(u_{\mathrm{Lu}}^{\mathrm{V} 1}\right)-\omega_{\mathrm{V} 1} \sigma^{\mathrm{V} 1}\left(u_{\mathrm{Ld}}^{\mathrm{V} 1}\right)+q \xi_{\mathrm{Tp}}^{\mathrm{V} 1}(t), \\
\tau \frac{d u_{\mathrm{Rp}}^{\mathrm{V} 1}=}{d t} & -u_{\mathrm{Rp}}^{\mathrm{V} 1}+h^{\mathrm{V} 1}+S_{\mathrm{Rp}}(t)-v_{\mathrm{Rp}}^{\mathrm{V} 1}-\omega_{\mathrm{V} 1} \sigma^{\mathrm{V} 1}\left(u_{\mathrm{Ru}}^{\mathrm{V} 1}\right)-\omega_{\mathrm{V} 1} \sigma^{\mathrm{V} 1}\left(u_{\mathrm{Rd}}^{\mathrm{V} 1}\right) \\
& -\omega_{\mathrm{V} 1} \sigma^{\mathrm{V} 1}\left(u_{\mathrm{Br}}^{\mathrm{V} 1}\right)-\omega_{\mathrm{V} 1} \sigma^{\mathrm{V} 1}\left(u_{\mathrm{B} 1}^{\mathrm{V} 1}\right)-\omega_{\mathrm{V} 1} \sigma^{\mathrm{V} 1}\left(u_{\mathrm{Tr}}^{\mathrm{V} 1}\right)-\omega_{\mathrm{V} 1} \sigma^{\mathrm{V} 1}\left(u_{\mathrm{T} 1}^{\mathrm{V} 1}\right)+q \xi_{\mathrm{Rp}}^{\mathrm{V} 1}(t),
\end{aligned}
$$

and

$$
\begin{aligned}
\tau \frac{d u_{\mathrm{Lp}}^{\mathrm{V} 1}}{d t}= & -u_{\mathrm{Lp}}^{\mathrm{V} 1}+h^{\mathrm{V} 1}+S_{\mathrm{Lp}}(t)-v_{\mathrm{Lp}}^{\mathrm{V} 1}-\omega_{\mathrm{V} 1} \sigma^{\mathrm{V} 1}\left(u_{\mathrm{Lu}}^{\mathrm{V} 1}\right)-\omega_{\mathrm{V} 1} \sigma^{\mathrm{V} 1}\left(u_{\mathrm{Ld}}^{\mathrm{V} 1}\right) \\
& -\omega_{\mathrm{V} 1} \sigma^{\mathrm{V} 1}\left(u_{\mathrm{Br}}^{\mathrm{V} 1}\right)-\omega_{\mathrm{V} 1} \sigma^{\mathrm{V} 1}\left(u_{\mathrm{B} 1}^{\mathrm{V} 1}\right)-\omega_{\mathrm{V} 1} \sigma^{\mathrm{V} 1}\left(u_{\mathrm{Tr}}^{\mathrm{V} 1}\right)-\omega_{\mathrm{V} 1} \sigma^{\mathrm{V} 1}\left(u_{\mathrm{T} 1}^{\mathrm{V} 1}\right)+q \xi_{\mathrm{Lp}}^{\mathrm{V} 1}(t) .
\end{aligned}
$$

The stochastic differential equations for the V1 motion detectors are

$$
\begin{aligned}
& \tau \frac{d u_{\mathrm{Br}}^{\mathrm{V} 1}}{d t}=-u_{\mathrm{Br}}^{\mathrm{V} 1}+h^{\mathrm{V} 1}+S_{\mathrm{Br}}(t)-v_{\mathrm{Br}}^{\mathrm{V} 1}-\omega_{\mathrm{V} 1} \sigma^{\mathrm{V} 1}\left(u_{\mathrm{Bp}}^{\mathrm{V} 1}\right)-\omega_{\mathrm{V} 1} \sigma^{\mathrm{V} 1}\left(u_{\mathrm{Rp}}^{\mathrm{V} 1}\right)-\omega_{\mathrm{V} 1} \sigma^{\mathrm{V} 1}\left(u_{\mathrm{Lp}}^{\mathrm{V} 1}\right)+q \xi_{\mathrm{Br}}^{\mathrm{V} 1}(t), \\
& \tau \frac{d u_{\mathrm{B} 1}^{\mathrm{V} 1}}{d t}=-u_{\mathrm{B} 1}^{\mathrm{V} 1}+h^{\mathrm{V} 1}+S_{\mathrm{B} 1}(t)-v_{\mathrm{B} 1}^{\mathrm{V} 1}-\omega_{\mathrm{V} 1} \sigma^{\mathrm{V} 1}\left(u_{\mathrm{Bp}}^{\mathrm{V} 1}\right)-\omega_{\mathrm{V} 1} \sigma^{\mathrm{V} 1}\left(u_{\mathrm{Rp}}^{\mathrm{V} 1}\right)-\omega_{\mathrm{V} 1} \sigma^{\mathrm{V} 1}\left(u_{\mathrm{Lp}}^{\mathrm{V} 1}\right)+q \xi_{\mathrm{B} 1}^{\mathrm{V} 1}(t), \\
& \tau \frac{d u_{\mathrm{Tr}}^{\mathrm{V} 1}}{d t}=-u_{\mathrm{Tr}}^{\mathrm{V} 1}+h^{\mathrm{V} 1}+S_{\mathrm{Tr}}(t)-v_{\mathrm{Tr}}^{\mathrm{V} 1}-\omega_{\mathrm{V} 1} \sigma^{\mathrm{V} 1}\left(u_{\mathrm{Tp}}^{\mathrm{V} 1}\right)-\omega_{\mathrm{V} 1} \sigma^{\mathrm{V} 1}\left(u_{\mathrm{Rp}}^{\mathrm{V} 1}\right)-\omega_{\mathrm{V} 1} \sigma^{\mathrm{V} 1}\left(u_{\mathrm{Lp}}^{\mathrm{V} 1}\right)+q \xi_{\mathrm{Tr}}^{\mathrm{V} 1}(t),
\end{aligned}
$$


APPENDIX (Continued)

$$
\begin{aligned}
& \tau \frac{d u_{\mathrm{T} 1}^{\mathrm{V} 1}}{d t}=-u_{\mathrm{T} 1}^{\mathrm{V} 1}+h^{\mathrm{V} 1}+S_{\mathrm{T} 1}(t)-v_{\mathrm{T} 1}^{\mathrm{V} 1}-\omega_{\mathrm{V} 1} \sigma^{\mathrm{V} 1}\left(u_{\mathrm{Tp}}^{\mathrm{V} 1}\right)-\omega_{\mathrm{V} 1} \sigma^{\mathrm{V} 1}\left(u_{\mathrm{Rp}}^{\mathrm{V} 1}\right)-\omega_{\mathrm{V} 1} \sigma^{\mathrm{V} 1}\left(u_{\mathrm{Lp}}^{\mathrm{V} 1}\right)+q \xi_{\mathrm{T} 1}^{\mathrm{V} 1}(t), \\
& \tau \frac{d u_{\mathrm{Ru}}^{\mathrm{V} 1}}{d t}=-u_{\mathrm{Ru}}^{\mathrm{V} 1}+h^{\mathrm{V} 1}+S_{\mathrm{Ru}}(t)-v_{\mathrm{Ru}}^{\mathrm{V} 1}-\omega_{\mathrm{V} 1} \sigma^{\mathrm{V} 1}\left(u_{\mathrm{Rp}}^{\mathrm{V} 1}\right)-\omega_{\mathrm{V} 1} \sigma^{\mathrm{V} 1}\left(u_{\mathrm{Bp}}^{\mathrm{V} 1}\right)-\omega_{\mathrm{V} 1} \sigma^{\mathrm{V} 1}\left(u_{\mathrm{Tp}}^{\mathrm{V} 1}\right)+q \xi_{\mathrm{Ru}}^{\mathrm{V} 1}(t), \\
& \tau \frac{d u_{\mathrm{Rd}}^{\mathrm{V} 1}}{d t}=-u_{\mathrm{Rd}}^{\mathrm{V} 1}+h^{\mathrm{V} 1}+S_{\mathrm{Rd}}(t)-v_{\mathrm{Rd}}^{\mathrm{V} 1}-\omega_{\mathrm{V} 1} \sigma^{\mathrm{V} 1}\left(u_{\mathrm{Rp}}^{\mathrm{V} 1}\right)-\omega_{\mathrm{V} 1} \sigma^{\mathrm{V} 1}\left(u_{\mathrm{Bp}}^{\mathrm{V} 1}\right)-\omega_{\mathrm{V} 1} \sigma^{\mathrm{V} 1}\left(u_{\mathrm{Tp}}^{\mathrm{V} 1}\right)+q \xi_{\mathrm{Rd}}^{\mathrm{V} 1}(t), \\
& \tau \frac{d u_{\mathrm{Lu}}^{\mathrm{V} 1}}{d t}=-u_{\mathrm{Lu}}^{\mathrm{V} 1}+h^{\mathrm{V} 1}+S_{\mathrm{Lu}}(t)-v_{\mathrm{Lu}}^{\mathrm{V} 1}-\omega_{\mathrm{V} 1} \sigma^{\mathrm{V} 1}\left(u_{\mathrm{Lp}}^{\mathrm{V} 1}\right)-\omega_{\mathrm{V} 1} \sigma^{\mathrm{V} 1}\left(u_{\mathrm{Bp}}^{\mathrm{V} 1}\right)-\omega_{\mathrm{V} 1} \sigma^{\mathrm{V} 1}\left(u_{\mathrm{Tp}}^{\mathrm{V} 1}\right)+q \xi_{\mathrm{Lu}}^{\mathrm{V} 1}(t),
\end{aligned}
$$

and

$$
\tau \frac{d u_{\mathrm{Ld}}^{\mathrm{V} 1}}{d t}=-u_{\mathrm{Ld}}^{\mathrm{V} 1}+h^{\mathrm{V} 1}+S_{\mathrm{Ld}}(t)-v_{\mathrm{Ld}}^{\mathrm{V} 1}-\omega_{\mathrm{V} 1} \sigma^{\mathrm{V} 1}\left(u_{\mathrm{Lp}}^{\mathrm{V} 1}\right)-\omega_{\mathrm{V} 1} \sigma^{\mathrm{V} 1}\left(u_{\mathrm{Bp}}^{\mathrm{V} 1}\right)-\omega_{\mathrm{V} 1} \sigma^{\mathrm{V} 1}\left(u_{\mathrm{Tp}}^{\mathrm{V} 1}\right)+q \xi_{\mathrm{Ld}}^{\mathrm{V} 1}(t) .
$$

The dynamics of the pattern-level (MT) detectors are described below. The rate of change of activation variable $u_{i}^{\mathrm{MT}}$ is determined by the time scale of activation change, $\tau$, the no-stimulus resting level, $h^{\mathrm{MT}}$, the feedforward of positive activation from local level (V1) detectors, $\left[u_{i}^{\mathrm{V} 1}\right]^{+}$, the adaptation level, $v_{i}^{\mathrm{MT}}$, additive Gaussian white noise, $\xi(t)$, with strength $q$, as well as by interactions from other pattern-level detectors. $\sigma^{\mathrm{MT}}\left(u_{j}^{\mathrm{MT}}\right)$ denotes the inhibitory coupling with other motion detectors with orthogonal directional selectivity, with $\omega_{\mathrm{s}}$ denoting the maximum strength of the coupling for detectors stimulated during the same frame (pattern-forming interactions), and $\omega_{\mathrm{n}}$ denoting the strength of inhibition of currently stimulated detectors on the activation of detectors that will be stimulated during the next frame (future-shaping interactions).

The stochastic differential equations for the MT motion detectors are

$$
\begin{aligned}
& \tau \frac{d u_{\mathrm{Br}}^{\mathrm{MT}}}{d t}=-u_{\mathrm{Br}}^{\mathrm{MT}}+h^{\mathrm{MT}}+\left[u_{\mathrm{Br}}^{\mathrm{V} 1}\right]^{+}-v_{\mathrm{Br}}^{\mathrm{MT}}-\omega_{\mathrm{s}} \sigma^{\mathrm{MT}}\left(u_{\mathrm{Lu}}^{\mathrm{MT}}\right) \\
& -\omega_{\mathrm{s}} \sigma^{\mathrm{MT}}\left(u_{\mathrm{Rd}}^{\mathrm{MT}}\right)-\omega_{\mathrm{n}} \sigma^{\mathrm{MT}}\left(u_{\mathrm{Ld}}^{\mathrm{MT}}\right)-\omega_{\mathrm{n}} \sigma^{\mathrm{MT}}\left(u_{\mathrm{Ru}}^{\mathrm{MT}}\right)+q \xi_{\mathrm{Br}}^{\mathrm{MT}}(t), \\
& \tau \frac{d u_{\mathrm{Bl}}^{\mathrm{MT}}}{d t}=-u_{\mathrm{Bl}}^{\mathrm{MT}}+h^{\mathrm{MT}}+\left[u_{\mathrm{Bl}}^{\mathrm{V} 1}\right]^{+}-v_{\mathrm{Bl}}^{\mathrm{MT}}-\omega_{\mathrm{s}} \sigma^{\mathrm{MT}}\left(u_{\mathrm{Ru}}^{\mathrm{MT}}\right) \\
& -\omega_{\mathrm{s}} \sigma^{\mathrm{MT}}\left(u_{\mathrm{Ld}}^{\mathrm{MT}}\right)-\omega_{\mathrm{n}} \sigma^{\mathrm{MT}}\left(u_{\mathrm{Rd}}^{\mathrm{MT}}\right)-\omega_{\mathrm{n}} \sigma^{\mathrm{MT}}\left(u_{\mathrm{Lu}}^{\mathrm{MT}}\right)+q \xi_{\mathrm{Bl}}^{\mathrm{MT}}(t), \\
& \tau \frac{d u_{\mathrm{Tr}}^{\mathrm{MT}}}{d t}=-u_{\mathrm{Tr}}^{\mathrm{MT}}+h^{\mathrm{MT}}+\left[u_{\mathrm{Tr}}^{\mathrm{V} 1}\right]^{+}-v_{\mathrm{Tr}}^{\mathrm{MT}}-\omega_{\mathrm{s}} \sigma^{\mathrm{MT}}\left(u_{\mathrm{Ru}}^{\mathrm{MT}}\right) \\
& -\omega_{\mathrm{s}} \sigma^{\mathrm{MT}}\left(u_{\mathrm{Ld}}^{\mathrm{MT}}\right)-\omega_{\mathrm{n}} \sigma^{\mathrm{MT}}\left(u_{\mathrm{Rd}}^{\mathrm{MT}}\right)-\omega_{\mathrm{n}} \sigma^{\mathrm{MT}}\left(u_{\mathrm{Lu}}^{\mathrm{MT}}\right)+q \xi_{\mathrm{Tr}}^{\mathrm{MT}}(t), \\
& \tau \frac{d u_{\mathrm{Tl}}^{\mathrm{MT}}}{d t}=-u_{\mathrm{Tl}}^{\mathrm{MT}}+h^{\mathrm{MT}}+\left[u_{\mathrm{Tl}}^{\mathrm{V} 1}\right]^{+}-v_{\mathrm{Tl}}^{\mathrm{MT}}-\omega_{\mathrm{s}} \sigma^{\mathrm{MT}}\left(u_{\mathrm{Lu}}^{\mathrm{MT}}\right) \\
& -\omega_{\mathrm{s}} \sigma^{\mathrm{MT}}\left(u_{\mathrm{Rd}}^{\mathrm{MT}}\right)-\omega_{\mathrm{n}} \sigma^{\mathrm{MT}}\left(u_{\mathrm{Ld}}^{\mathrm{MT}}\right)-\omega_{\mathrm{n}} \sigma^{\mathrm{MT}}\left(u_{\mathrm{Ru}}^{\mathrm{MT}}\right)+q \xi_{\mathrm{Tl}}^{\mathrm{MT}}(t), \\
& \tau \frac{d u_{\mathrm{Ru}}^{\mathrm{MT}}}{d t}=-u_{\mathrm{Ru}}^{\mathrm{MT}}+h^{\mathrm{MT}}+\left[u_{\mathrm{Ru}}^{\mathrm{V} 1}\right]^{+}-v_{\mathrm{Ru}}^{\mathrm{MT}}-\omega_{\mathrm{s}} \sigma^{\mathrm{MT}}\left(u_{\mathrm{Bl}}^{\mathrm{MT}}\right) \\
& -\omega_{\mathrm{s}} \sigma^{\mathrm{MT}}\left(u_{\mathrm{Tr}}^{\mathrm{MT}}\right)-\omega_{\mathrm{n}} \sigma^{\mathrm{MT}}\left(u_{\mathrm{Br}}^{\mathrm{MT}}\right)-\omega_{\mathrm{n}} \sigma^{\mathrm{MT}}\left(u_{\mathrm{Tl}}^{\mathrm{MT}}\right)+q \xi_{\mathrm{Ru}}^{\mathrm{MT}}(t), \\
& \tau \frac{d u_{\mathrm{Rd}}^{\mathrm{MT}}}{d t}=-u_{\mathrm{Rd}}^{\mathrm{MT}}+h^{\mathrm{MT}}+\left[u_{\mathrm{Rd}}^{\mathrm{V} 1}\right]^{+}-v_{\mathrm{Rd}}^{\mathrm{MT}}-\omega_{\mathrm{s}} \sigma^{\mathrm{MT}}\left(u_{\mathrm{Br}}^{\mathrm{MT}}\right) \\
& -\omega_{\mathrm{s}} \sigma^{\mathrm{MT}}\left(u_{\mathrm{Tl}}^{\mathrm{MT}}\right)-\omega_{\mathrm{n}} \sigma^{\mathrm{MT}}\left(u_{\mathrm{Bl}}^{\mathrm{MT}}\right)-\omega_{\mathrm{n}} \sigma^{\mathrm{MT}}\left(u_{\mathrm{Tr}}^{\mathrm{MT}}\right)+q \xi_{\mathrm{Rd}}^{\mathrm{MT}}(t), \\
& \tau \frac{d u_{\mathrm{Lu}}^{\mathrm{MT}}}{d t}=-u_{\mathrm{Lu}}^{\mathrm{MT}}+h^{\mathrm{MT}}+\left[u_{\mathrm{Lu}}^{\mathrm{V} 1}\right]^{+}-v_{\mathrm{Lu}}^{\mathrm{MT}}-\omega_{\mathrm{s}} \sigma^{\mathrm{MT}}\left(u_{\mathrm{Br}}^{\mathrm{MT}}\right) \\
& -\omega_{\mathrm{s}} \sigma^{\mathrm{MT}}\left(u_{\mathrm{Tl}}^{\mathrm{MT}}\right)-\omega_{\mathrm{n}} \sigma^{\mathrm{MT}}\left(u_{\mathrm{Bl}}^{\mathrm{MT}}\right)-\omega_{\mathrm{n}} \sigma^{\mathrm{MT}}\left(u_{\mathrm{Tr}}^{\mathrm{MT}}\right)+q \xi_{\mathrm{Lu}}^{\mathrm{MT}}(t),
\end{aligned}
$$

and

$$
\begin{aligned}
\tau \frac{d u_{\mathrm{Ld}}^{\mathrm{MT}}}{d t}= & -u_{\mathrm{Ld}}^{\mathrm{MT}}+h^{\mathrm{MT}}+\left[u_{\mathrm{Ld}}^{\mathrm{V} 1}\right]^{+}-v_{\mathrm{Ld}}^{\mathrm{MT}}-\omega_{\mathrm{s}} \sigma^{\mathrm{MT}}\left(u_{\mathrm{Bl}}^{\mathrm{MT}}\right) \\
& -\omega_{\mathrm{s}} \sigma^{\mathrm{MT}}\left(u_{\mathrm{Tr}}^{\mathrm{MT}}\right)-\omega_{\mathrm{n}} \sigma^{\mathrm{MT}}\left(u_{\mathrm{Br}}^{\mathrm{MT}}\right)-\omega_{\mathrm{n}} \sigma^{\mathrm{MT}}\left(u_{\mathrm{Tl}}^{\mathrm{MT}}\right)+q \xi_{\mathrm{Ld}}^{\mathrm{MT}}(t) .
\end{aligned}
$$




\section{APPENDIX (Continued)}

The implementation of adaptation is consistent with evidence that the mechanism of adaptation lies within the adapted neuron (Carandini \& Ferster, 1997; Sanchez-Vives, Nowak, \& McCormick, 2000). The adaptation variables, $v_{i}$, have an initial value of 0 , increase due to a positive, nonlinear function of activation, and have no noise added; therefore, they can have only positive values. The dynamics of the adaptation variables are described by

$$
\tau^{\alpha} \frac{d v_{i}}{d t}=-v_{i}+\alpha \sigma^{\text {adapt }}\left(u_{i}\right)
$$

where $\sigma^{\text {adapt }}(u)$ is the Naka-Rushton (1966) nonlinearity illustrated in Figure 5A. Its equation is

$$
\sigma^{\text {adapt }}(u)=\frac{(u-1.5)^{0.5}}{(10-1.5)^{0.5}+(u-1.5)^{0.5}} \text { for } u \geq-1.5 ; \sigma^{\text {adapt }}(u)=0 \text { for } u<-1.5 \text {. }
$$

Local-level (V1) inhibitory interactions among activational variables representing detectors responsive to motion-independent spatial information and detectors responsive to motion-specifying stimulus information are characterized by the Naka-Rushton (1966) nonlinearity illustrated in Figure 5B. Its equation is

$$
\sigma^{\mathrm{V} 1}(u)=\frac{(u-0.5)^{20}}{(0-0.5)^{20}+(u-0.5)^{20}} \text { for } u \geq-0.5 ; \sigma^{\mathrm{V} 1}(u)=0 \text { for } u<-0.5 \text {. }
$$

Pattern-level (MT) inhibitory interactions among activational variables representing motion detectors with orthogonal directional selectivity are characterized by the Naka-Rushton (1966) nonlinearity illustrated in Figure $5 \mathrm{C}$. Its equation is

$$
\sigma^{\mathrm{MT}}(u)=\frac{(u-1.5)^{0.5}}{(10-1.5)^{0.5}+(u-1.5)^{0.5}} \text { for } u \geq-1.5 ; \sigma^{\mathrm{MT}}(u)=0 \text { for } u<-1.5 .
$$

The feedforward input from local-level motion detectors to pattern-level motion direction variables is halfwave rectified, as follows:

$$
[u]^{+}=\max (u, 0) .
$$

Because of the back-and-forth nature of motion for the motion quartet, four of the eight motion directions (Ld, $\mathrm{Tr}, \mathrm{Ru}$, and $\mathrm{Bl}$ ) are stimulated during odd-numbered frames, and the other four motion directions ( $\mathrm{Lu}, \mathrm{Tl}, \mathrm{Rd}$, and $\mathrm{Br}$ ) are stimulated during even-numbered frames. The functions $S_{i}(t)$ representing the time courses of stimulusinitiated activation, therefore, are approximated by square waves that alternate on and off every $200 \mathrm{msec}$ and that are $180^{\circ}$ out of phase for the motions stimulated during odd- and even-numbered frames. With the exception of the frames during which there is a perturbation, the stimulus-initiated activation values (which are indicated in the text) are the same in all three experiments.

All simulations are based on a single set of parameters: $\tau=20 \mathrm{msec}, \tau^{\alpha}=2,000 \mathrm{msec}, h^{\mathrm{V} 1}=-5, h^{\mathrm{MT}}=$ $-3, q=0.15, \omega_{\mathrm{V} 1}=2, \omega_{\mathrm{s}}=25, \omega_{\mathrm{n}}=2$, and $\alpha=1.8$. 